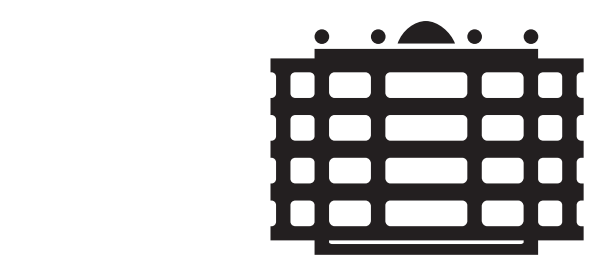

TECHNISCHE UNIVERSITÄT

CHEMNITZ

\title{
Embedded in the Shadow of the Separator
}
F. Göring
C. Helmberg
M. Wappler

Preprint 2005-12

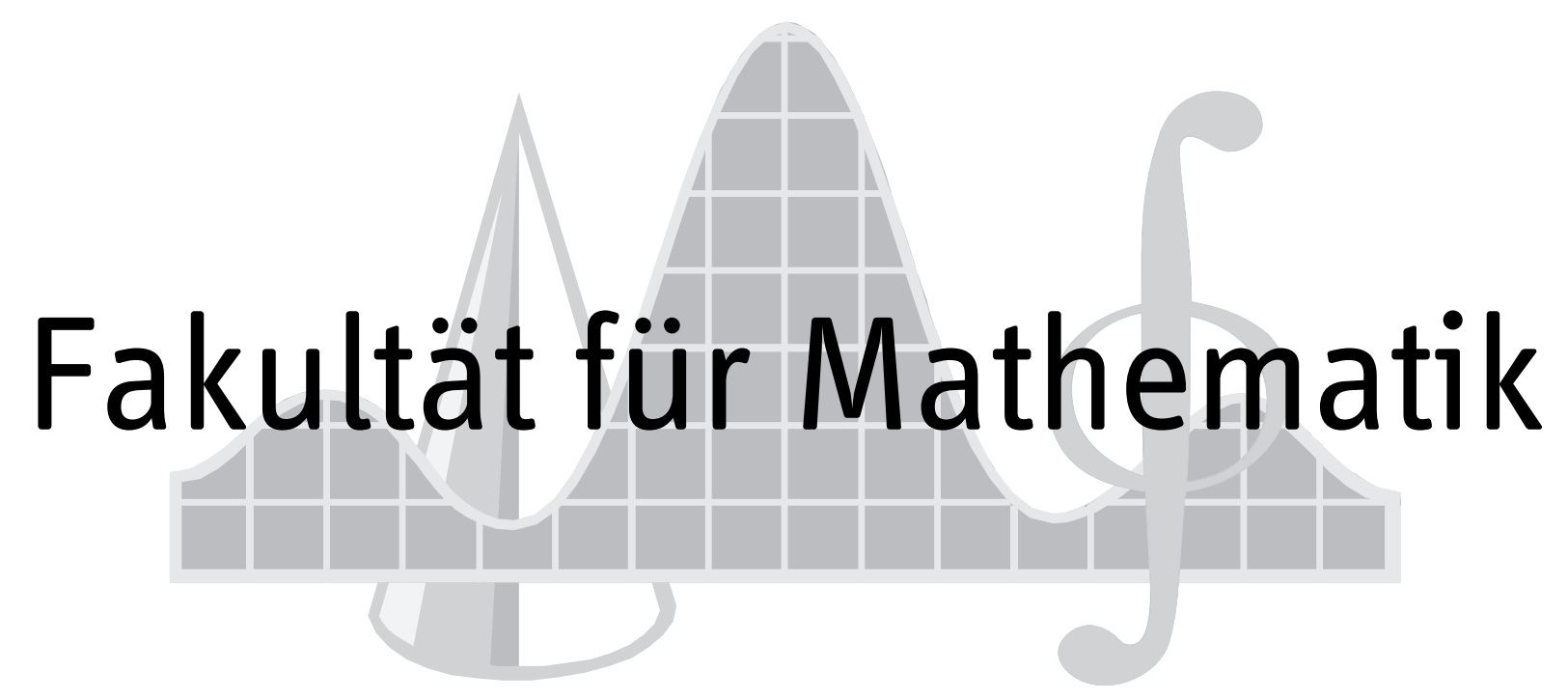


Impressum:

Herausgeber:

Der Dekan der

Fakultät für Mathematik

an der Technischen Universität Chemnitz

Sitz:

Reichenhainer Straße 41

09126 Chemnitz

Postanschrift:

09107 Chemnitz

Telefon: (0371) 531-2662

Telefax: (0371) 531-4947

E-Mail: dekanat@mathematik.tu-chemnitz.de

Internet:

http://www.tu-chemnitz.de/mathematik/

ISSN 1614-8835 (Print) 


\title{
Embedded in the Shadow of the Separator
}

\author{
Frank Göring, Christoph Helmberg, and Markus Wappler*
}

September 2, 2005

\begin{abstract}
We study the problem of maximizing the second smallest eigenvalue of the Laplace matrix of a graph over all nonnegative edge weightings with bounded total weight. The optimal value is the absolute algebraic connectivity introduced by Fiedler, who proved tight connections of this value to the connectivity of the graph. Using semidefinite programming techniques and exploiting optimality conditions we show that the problem is equivalent to finding an embedding of the $n$ nodes in $n$-space so that their barycenter is at the origin, the distance between adjacent nodes is bounded by one and the nodes are spread as much as possible (the sum of the squared norms is maximized). For connected graphs we prove that for any separator in the graph, at least one of the two separated node sets is embedded in the shadow (with the origin being the light source) of the convex hull of the separator. In particular, the barycenters of partitions induced by separators are separated by the affine subspace spanned by the nodes of the separator. Furthermore, we show that there always exists an optimal embedding whose dimension is bounded by the tree width of the graph plus one.

Keywords: spectral graph theory, semidefinite programming, eigenvalue optimization, embedding, graph partitioning, tree-width
\end{abstract}

MSC 2000: 05C50; 90C22, 90C35, 05C10, 05C78

\section{Introduction}

Let $G=(N, E)$ be an undirected graph with node set $N=\{1, \ldots, n\}$ and edge set $E \subseteq\{\{i, j\}: i, j \in N, i \neq j\}$. Edge $\{i, j\}$ will be abbreviated by $i j$ if there is no danger of confusion. The adjacency matrix $A \in \mathbb{R}^{n \times n}$ of the graph is defined as the (symmetric) matrix having $a_{i j}=1$ if $i j \in E$ and 0 otherwise. The Laplace matrix or Laplacian of the graph is the matrix $L=\operatorname{diag}(A e)-A$, where $e$ denotes the vector of all ones of appropriate dimension and $\operatorname{diag}(v)$ denotes the diagonal matrix having $v$ on its main diagonal. For symmetric matrices $H \in \mathbb{R}^{n \times n}$ we order the eigenvalues by $\lambda_{1}(H) \leq \lambda_{2}(H) \leq \cdots \leq \lambda_{n}(H)$.

*Fakultät für Mathematik, Technische Universität Chemnitz, D-09107 Chemnitz, Germany. \{frank.goering, helmberg, markus.wappler\}@mathematik.tu-chemnitz.de 
Because the Laplacian $L$ is positive semidefinite and $L e=0$, we have $\lambda_{1}(L)=0$ with eigenvector $e$. Fiedler $[6,7]$ showed that the second smallest eigenvalue $\lambda_{2}(L)$ is tightly related to edge and vertex connectivity of the graph. In particular, $\lambda_{2}(G)$ is positive if and only if $G$ is connected. Therefore, Fiedler called $\lambda_{2}(L)$ the algebraic connectivity of the graph. Eigenvectors to $\lambda_{2}(L)$, often referred to as Fiedler vectors, have been used quite successfully in heuristics for graph partitioning in parallel computing [21, 22, 15], in clustering of geometric objects [2] or hyperlinks in the world wide web [13] or even computer vision [16]. The second smallest eigenvalue allows to derive various bounds in graph partitioning or bandwidth optimization $([14,12])$; further properties of the Laplacian spectrum are presented in $[8,9,10,3,26]$ and $[18,19]$ give a survey on the Laplacian spectrum of graphs. See also $[4,20]$ for related applications of spectral graph theory in combinatorial optimization.

By the Courant-Fischer Theorem, the eigenvalue $\lambda_{2}(L)$ and its eigenvectors may be characterized as optimal solutions to the optimization problem

$$
\lambda_{2}(L)=\min _{v \in \mathbb{R}^{n}, v^{T} e=0,\|v\|=1} v^{T} L v
$$

The usefulness of $\lambda_{2}$ and its eigenvectors in graph partitioning should relate to this characterization in some way. In order to get a better understanding of these connections, it seems natural to study weighted matrices on the same support (i.e., arc weighted graphs on the same edge set) that are extremal in the sense, that for their distribution of the weight, $\lambda_{2}$ is maximal. We will investigate this problem, already raised by Fiedler [7] under the name "absolute algebraic connectivity", within the setting of semidefinite programming. It turns out that the dual to this problem may be interpreted as an embedding problem of the nodes in $\mathbb{R}^{n}$, see (4). We show that optimal embeddings have structural properties tightly connected to separators in graphs (Th. 12). In particular, if a subset $S \subset N$ of nodes separates the graph into two disconnected node sets $K_{1}, K_{2}$ forming a partition of $N \backslash S$, then for one of the two sets, say $K_{1}$, all nodes are in the "shadow" of the convex hull of the nodes in $S$ as seen from the origin, i.e., the straight line segment between any node of $K_{1}$ and the origin intersects the convex hull of $S$. This offers good geometric insight into the usefulness of Fiedler vectors for graph partitioning. In addition, Th. 12 yields via Cor. 30 a dual geometric interpretation for the result of Fiedler [8] on the "absolute algebraic connectivity" for trees.

The embedding may also be interpreted as a variant of vector labelings of graphs as introduced in [17] for the Lovász $\vartheta$-number of a graph. On first sight, strong similarities exist with respect to the Colin de Verdière number $\mu(G)$, see the excellent survey [24]. But the strong Arnold-property is not required in our context, so no direct connection to $\mu(G)$ should be expected. Yet, similar to maximizing the corank in the Colin de Verdière number, one may ask for an optimal embedding of minimal dimension. Even though we are still far from answering this question to our full satisfaction, we are able to exhibit an intriguing bound based on the tree width of the graph. Indeed, we show in the proof of Th. 19, that there is always an optimal embedding whose dimension is bounded by the cardinality of a "central" node of an arbitrary tree decomposition of $G$. This bound is 
tight for some particular graph classes (see Ex. 22). None the less, the bound seems to be far too pessimistic, e.g., for planar graphs. Therefore it is conceivable that significantly better bounds can be obtained by minor related approaches.

The paper is organized as follows. In Section 2 the embedding problem is derived as the dual problem to the eigenvalue optimization problem of determining the absolute algebraic connectivity. Section 2 introduces several basic geometric operations on embeddings that will later be used to improve the objective or to reduce the dimension of a given embedding. Section 4 holds the core of the paper as it gives the main theorem on the shadow of the separator and the bound on the minimal dimension over all optimal embeddings. Finally, Section 5 concludes with an example of a class of graphs, for which the bound on the dimension is attained, and with special structural properties of optimal embeddings in the presence of articulation points, which includes the case of trees.

We use basic notions and notation from graph theory and semidefinite programming ([25]). In particular, the (unweighted) distance of two nodes in a (connected) graph is the minimum number of edges in a path connecting the two nodes; for symmetric $H \in \mathbb{R}^{n \times n}$, $H \succeq 0$ is used to denote positive semidefiniteness; for matrices $A, B \in \mathbb{R}^{m \times n},\langle A, B\rangle=$ $\sum_{i j} A_{i j} B_{i j}$ is the canonical inner product; $\|\cdot\|$ refers to the usual Euclidean norm; $e$ denotes the vector of all ones of appropriate size; $p_{C}(\cdot)$ is the projection on a closed convex set $C$.

\section{Formulation as an Optimal Embedding Problem}

In the remainder of the paper we assume, that the graph $G=(N, E)$ is connected and $n \geq 2$. Let

$$
\mathcal{W}=\left\{w \in \mathbb{R}_{+}^{E}: \sum_{i j \in E} w_{i j}=w^{T} e \leq 1\right\}
$$

denote the set of all possible nonnegative edge weightings that sum up to at most 1 . For a particular $w \in \mathcal{W}$, let $A_{w}$ denote the weighted adjacency matrix, i.e., $A_{i j}=w_{i j}$ for $i j \in E$ and 0 otherwise, and $L_{w}=\operatorname{diag}\left(A_{w} e\right)-A_{w}$ the corresponding weighted Laplacian. For $i, j \in V, i \neq j$ define $E_{i j} \in \mathbb{R}^{n \times n}$ as the matrix having the two diagonal elements $\left(E_{i j}\right)_{i i}=\left(E_{i j}\right)_{j j}=1$, the two offdiagonal elements $\left(E_{i j}\right)_{i j}=\left(E_{i j}\right)_{j i}=-1$ and all other elements equal to zero. Then we may rewrite the Laplacian as

$$
L_{w}=\sum_{i j \in E} w_{i j} E_{i j}
$$

The matrix $L_{w}$ is positive semidefinite (because $E_{i j}$ is positive semidefinite and $w_{i j} \geq 0$ for all $i j \in E$ ) and has an eigenvalue zero with eigenvector $e$ (because $E_{i j} e=0$ ). Our basic optimization problem reads

$$
\max _{w \in \mathcal{W}} \lambda_{2}\left(L_{w}\right)
$$

Since $G$ is assumed to be connected, the result of Fiedler [6] for $w=\frac{1}{|E|} e$ asserts $\lambda_{2}\left(L_{w}\right)=$ $\frac{1}{|E|} \lambda_{2}(L)>0$, so the optimum value is strictly positive. In order to reformulate the optimization problem as a semidefinite program it will be convenient to shift the smallest 
eigenvalue 0 to a sufficiently large value. Thus, (1) may be rewritten as the following semidefinite program,

$$
\begin{array}{cl}
\max & \lambda \\
\text { s.t. } & \sum_{i j \in E} w_{i j} E_{i j}+\mu e e^{T}-\lambda I \succeq 0 \\
& \sum_{i j \in E} w_{i j} \leq 1 \\
& w \geq 0, \lambda, \mu \text { free, }
\end{array}
$$

Because the optimal value is strictly greater than zero by the connectedness of $G$, we may rescale the problem by $1 / \lambda$ and equivalently minimize the sum of the weights instead,

$$
\begin{array}{cl}
\min & \sum_{i j \in E} w_{i j} \\
\mathrm{s.t.} & \sum_{i j \in E} w_{i j} E_{i j}+\mu e e^{T} \succeq I \\
& w \geq 0, \mu \text { free, }
\end{array}
$$

Note, that by the considerations above, $w=\frac{1}{\lambda_{2}(L)-\varepsilon} e, \mu=1+\varepsilon$ is a strictly feasible solution for $\lambda_{2}(L)>\varepsilon>0$. Therefore the program attains its optimal solution and semidefinite duality theory together with strict feasibility asserts that the optimal value of its dual semidefinite program is also attained. The dual reads

$$
\begin{aligned}
\max & \langle I, X\rangle \\
\text { s.t. } & \left\langle e e^{T}, X\right\rangle=0 \\
& \left\langle E_{i j}, X\right\rangle \leq 1 \quad \text { for } i j \in E \\
& X \succeq 0 .
\end{aligned}
$$

Now consider a Gram representation of $X$ via a matrix $V \in \mathbb{R}^{n \times n}$ with $X=V^{T} V$ and denote column $i$ of $V$ by $v_{i}, V=\left[v_{1}, \ldots, v_{n}\right]$. Then

$$
X_{i j}=v_{i}^{T} v_{j} \quad \text { and } \quad\left\langle E_{i j}, X\right\rangle=\left\|v_{i}\right\|^{2}-2 v_{i}^{T} v_{j}+\left\|v_{j}\right\|^{2}=\left\|v_{i}-v_{j}\right\|^{2} .
$$

Since $0=\left\langle e e^{T}, X\right\rangle=e^{T} X e=e^{T} V^{T} V e$ or $V e=\sum v_{i}=0$, the dual semidefinite program (3) translates directly to

$$
\begin{aligned}
\max & \sum_{i \in N}\left\|v_{i}\right\|^{2} \\
\text { s.t. } & \left(\sum_{i \in N} v_{i}\right)^{2}=0 \\
& \left\|v_{i}-v_{j}\right\|^{2} \leq 1 \quad \text { for } i j \in E \\
& v_{i} \in \mathbb{R}^{n} \text { for } i \in N .
\end{aligned}
$$

Thus, the dual problem to (1) is equivalent to finding an embedding of the nodes of the graph in $n$-space so that their barycenter is at the origin (we will call this the equilibrium constraint), the distances of adjacent nodes are bounded by one, and the sum of their squared norms is maximized. 


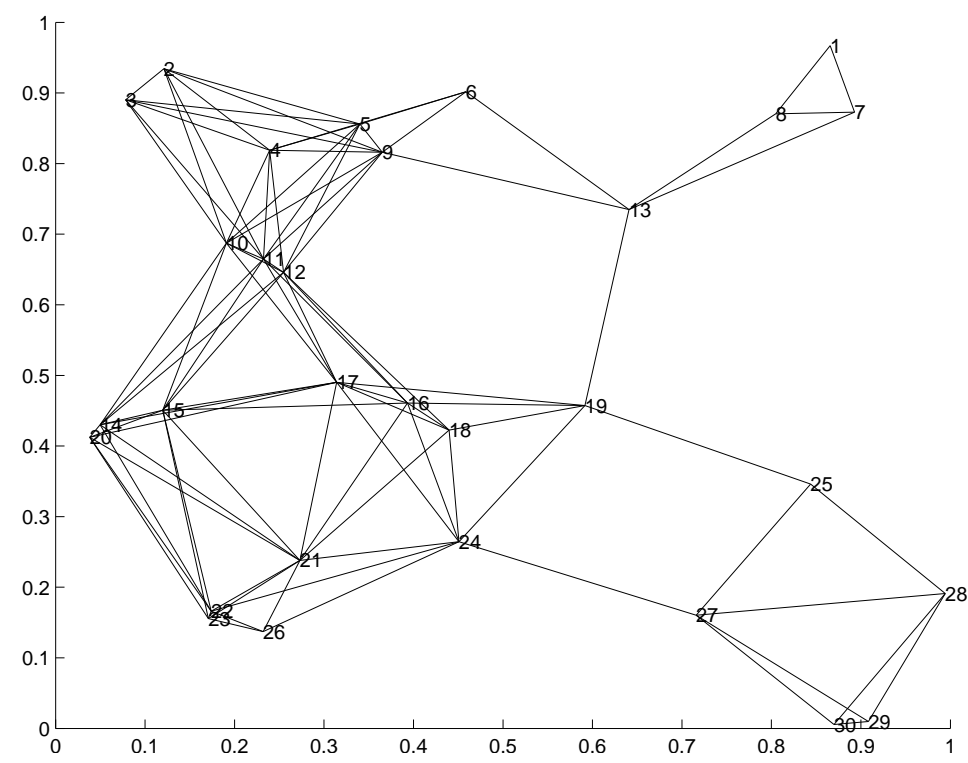

Figure 1: Original graph: The 30 vertices, picked randomly in $[0,1]^{2}$, are connected by an edge if the Euclidean distance is at most 0.3 .

Remark 1 Together with the KKT conditions (we do not list feasibility constraints again)

$$
\begin{aligned}
& v_{j}+\sum_{i j \in E} w_{i j}\left(v_{i}-v_{j}\right)+\mu \sum_{i \in N} v_{i}=0 \quad \forall j \in N \\
& w_{i j}\left(1-\left\|v_{i}-v_{j}\right\|^{2}\right)=0 \quad \forall i j \in E \\
& \mu\left(\sum_{i \in N} v_{i}\right)^{2}=0
\end{aligned}
$$

the embedding problem suggests the following physical interpretation of optimal primal and dual solutions. Consider each node as having a point mass of unit size and imagine each edge being a mass free rope of length one that connects the points. Now the optimum solution of (4) corresponds to an equilibrium solution of this net spread within a force field that acts with force $v$ on a point of mass one at position $v$. The $w_{i j}$ are the forces acting along rope $i j$. Indeed, all $w_{i j}>0$ are on the same scale as the force field, because $w_{i j}>0$ only if $\left\|v_{i}-v_{j}\right\|^{2}=1$. So the first line of the KKT conditions asserts that these forces are in equilibrium in each point ( $\mu \sum v_{i}=0$ by feasibility, so this term does not enter). If an optimal two dimensional embedding exists, such a physical situation is encountered when spreading the net on a disk rotating around its center (the centripetal force is $m \omega^{2} r$, where $m$ is the mass, $\omega$ the angular frequency and $r$ the radius).

We illustrate the embedding for a graph on 30 vertices. It was generated by picking the vertices randomly in the unit square and by connecting two points by an edge if their Euclidean distance is at most 0.3. The original graph is displayed in Fig. 1. The edge 


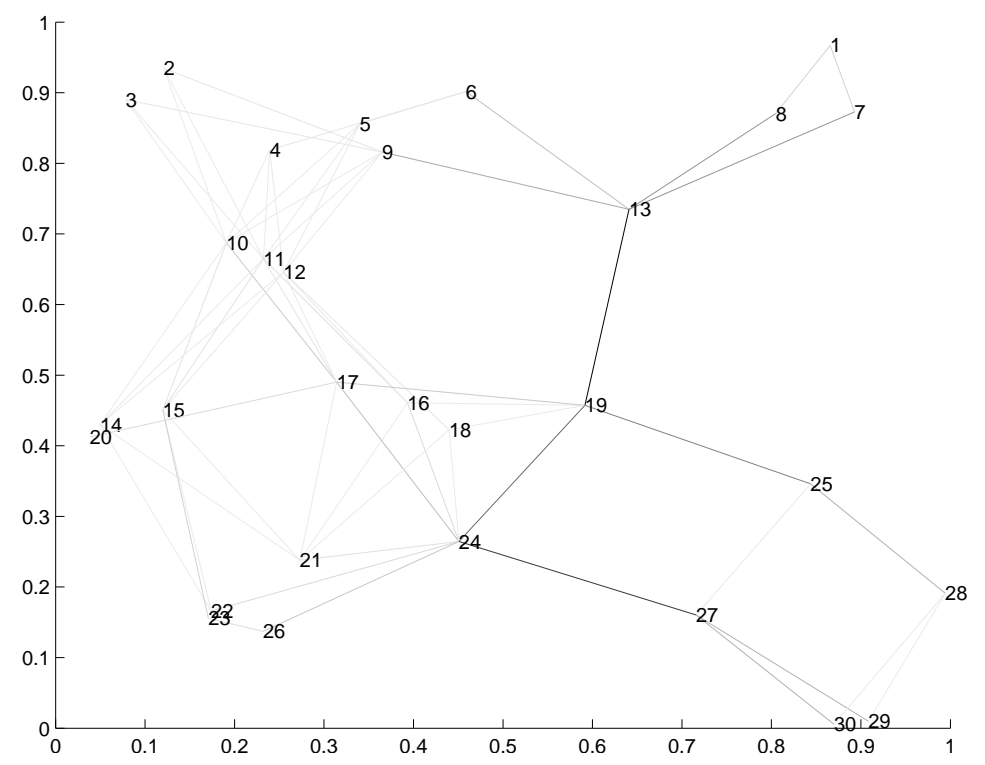

Figure 2: Graph with optimal edge weights.

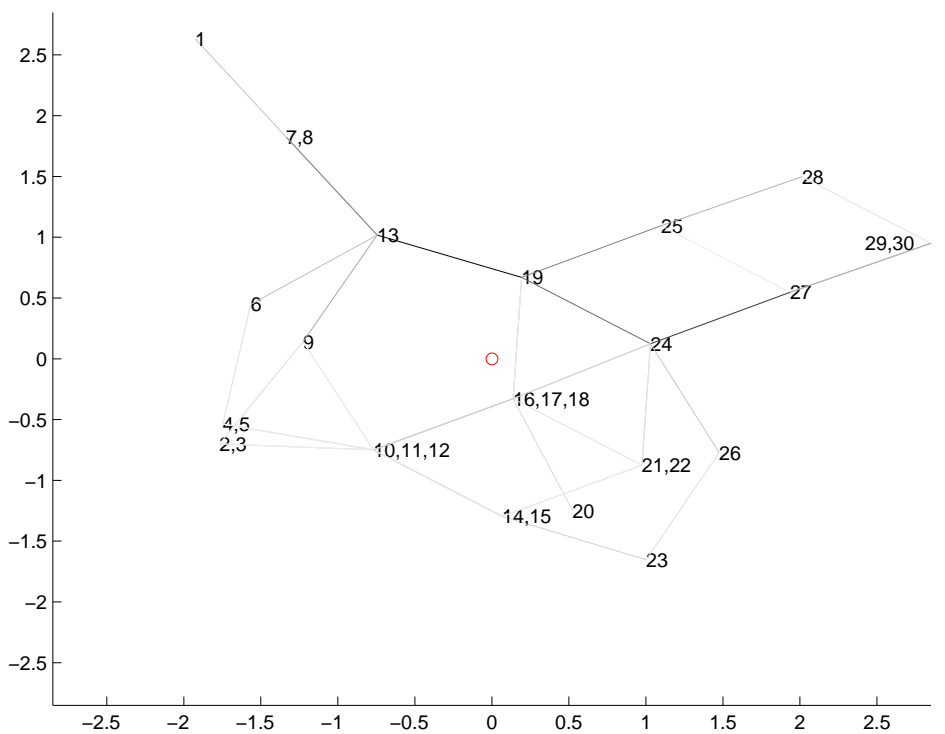

Figure 3: Optimal embedding (the central circle indicates the origin). 
weights corresponding to an optimal solution of problem (2) are given in grey shades in Fig. 2 (white is weight 0 , black is maximum weight). The optimal embedding corresponding to (4) was computed using SeDuMi [23] and is displayed in Fig. 2 (in fact, the optimal embedding is indeed two dimensional in this case; the origin is indicated by the small circle in the center). Note, that embedding coordinates are identical for several nodes of the original graph. We also point out that in the optimal embedding the block formed by the nodes $\{1,7,8,13\}$ with articulation point 13 is stretched right out along the ray emanating from the origin and passing through the articulation point.

Remark 2 The interpretation of the $w_{i j}$ as Lagrange multipliers to the embedding problem (4) may also turn out to be useful in judging the importance of certain links in networks (e.g. in telecommunication). Indeed, the size of $w_{i j}$ indicates the marginal increase in distance of the points in the network, if the length of the chord connecting $i$ and $j$ (see Rem. 1) is increased, or just dropped. Thus loosing a link ij with a large weight $w_{i j}$ may lead to a significant increase in transmission length or significant additional traffic on several other edges.

\section{Operations on Point Sets in the Embedding}

In order to describe structural properties of optimal embeddings of (4) we first study basic movements on subsets of points and their effect on the cost function. We start by observing that the rotation of a block of vectors around its barycenter does not affect the value of the cost function (we do not yet bother about feasibility).

Observation 3 (Rotation around the Barycenter) Let $v_{i} \in \mathbb{R}^{n}$ for $i \in K \subseteq N$ with $\bar{v}=\frac{1}{|K|} \sum_{i \in K} v_{i}$ their barycenter. If $v_{i}^{\prime} \in \mathbb{R}^{n}, i \in K$, result from rotation or reflection of the $v_{i}$ at $\bar{v}$ then $\sum_{i \in K}\left\|v_{i}^{\prime}\right\|^{2}=\sum_{i \in K}\left\|v_{i}\right\|^{2}$.

Proof. For $i \in K$ we have $v_{i}^{\prime}=Q\left(v_{i}-\bar{v}\right)+\bar{v}$, where $Q^{\top} Q=Q Q^{\top}=I$. Therefore

$$
\begin{aligned}
\sum_{i \in K}\left\|v_{i}^{\prime}\right\|^{2} & =\sum_{i \in K}\left\|Q\left(v_{i}-\bar{v}\right)\right\|^{2}+2\langle Q \underbrace{\sum_{i \in K}\left(v_{i}-\bar{v}\right)}_{=0}, \bar{v}\rangle+|K|\|\bar{v}\|^{2} \\
& =\sum_{i \in K}\left\|v_{i}-\bar{v}\right\|^{2}+2\left\langle\sum_{i \in K}\left(v_{i}-\bar{v}\right), \bar{v}\right\rangle+|K|\|\bar{v}\|^{2} \\
& =\sum_{i \in K}\left\|v_{i}-\bar{v}+\bar{v}\right\|^{2} \\
& =\sum_{i \in K}\left\|v_{i}\right\|^{2} .
\end{aligned}
$$

Next, we analyze the uniform translation of a block of vectors. 


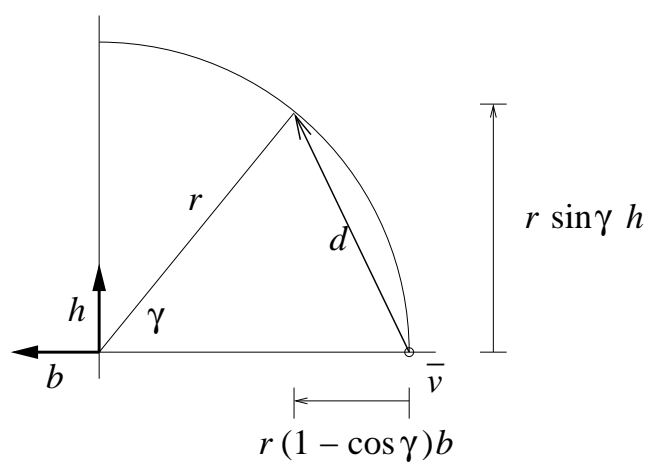

Figure 4: Rotation around an Affine Subspace.

Observation 4 (Translation) Let $d \in \mathbb{R}^{n}, v_{i} \in \mathbb{R}^{n}$ and $v_{i}^{\prime}=v_{i}+d$ for $i \in K \subseteq N$. Then $\sum_{i \in K}\left\|v_{i}^{\prime}\right\|^{2}=\sum_{i \in K}\left\|v_{i}\right\|^{2}+|K|\langle 2 \bar{v}+d, d\rangle$, where $\bar{v}=\frac{1}{|K|} \sum_{i \in K} v_{i}$ is the barycenter of the $v_{i}$.

Proof. $\sum_{i \in K}\left\|v_{i}+d\right\|^{2}=\sum_{i \in K}\left\|v_{i}\right\|^{2}+2\langle|K| \bar{v}, d\rangle+|K|\langle d, d\rangle$.

Thus, in a combined translation and rotation of a block of vectors, the change in the cost function can be determined from the translation of the barycenter of the block. For a block connected to the graph by an articulation point, a basic step to improve a given embedding might be to rotate the block outwards around the articulation point while keeping the distances within the block constant (for feasibility, the entire embedding will have to be recentered, too). In the proof of the main theorem we will need a particular type of rotation around a given affine subspace.

Observation 5 (Rotation around an Affine Subspace) Let $v_{i} \in \mathbb{R}^{n}$ for $i \in K \subseteq N$ with barycenter $\bar{v}=\frac{1}{|K|} \sum_{i \in K} v_{i}$ satisfy, for all $i \in K, h^{T} v_{i}=0$ and $b^{T} v_{i}<\beta$ for given $b, h \in \mathbb{R}^{n}, \beta>0$ with $\|b\|=\|h\|=1, b^{T} h=0$. Let $v_{i}^{\prime}$ arise from rotating the $v_{i}$ by angle $\gamma$ around the affine subspace $\mathcal{L}=\left\{x \in \mathbb{R}^{n}: h^{T} x=0, b^{T} x=\beta\right\}$, then

$$
\sum_{i \in K}\left\|v_{i}^{\prime}\right\|^{2}=\sum_{i \in K}\left\|v_{i}\right\|^{2}+2|K| r(1-\cos \gamma) \beta \quad \text { with } r=\beta-b^{T} \bar{v}>0 .
$$

Proof. By Obs. 3 the rotation around $L$ may be split into a rotation of the points in $K$ around $\bar{v}$ and a translation as analyzed in Obs. 4. The corresponding displacement $d$ for rotating $\bar{v}$ around $L$ by angle $\gamma$ is $d=r(\sin \gamma) h+r(1-\cos \gamma) b$ where $r=\beta-b^{T} \bar{v}>0$ is the radius (see Fig. 4). By Obs. $4, \bar{v}^{T} h=0$, and $b^{T} h=0$ the cost function changes by

$$
\begin{aligned}
|K|\left(2 \bar{v}^{T} d+d^{T} d\right) & =|K|\left(2 r(1-\cos \gamma) \bar{v}^{T} b+r^{2}\left[\sin ^{2} \gamma+(1-\cos \gamma)^{2}\right]\right) \\
& =|K|\left(2 r(1-\cos \gamma) \bar{v}^{T} b+r^{2}[2-2 \cos \gamma]\right) \\
& =2|K| r(1-\cos \gamma)\left(\bar{v}^{T} b+r\right) \\
& =2|K| r(1-\cos \gamma) \beta .
\end{aligned}
$$




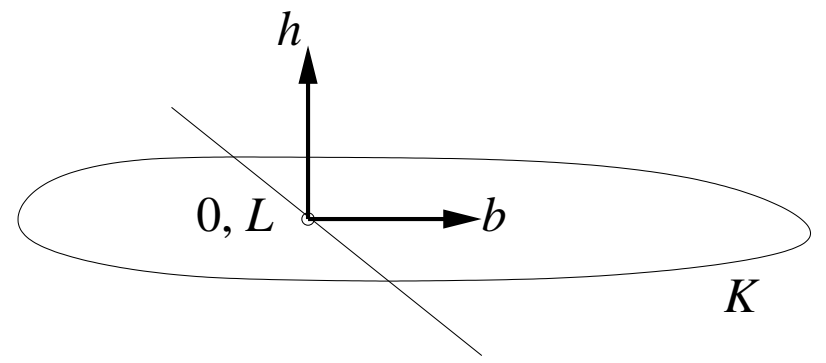

Figure 5: Initial setting before the transformation of $K$ in Obs. 6-8.

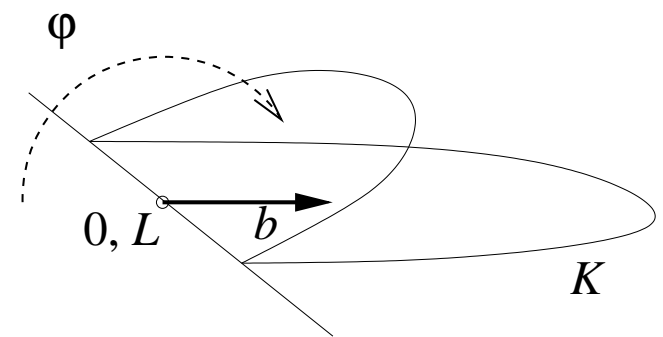

Figure 6: $\varphi$ folds $K$ into the halfspace specified by $b$ (Obs. 6).

Next, we construct a continuous transformation that collapses the points of a subset of nodes around a given subspace into a flat halfspace specified by the subspace and a direction $b$. During this transformation the cost function is kept constant and all distances between the points involved are not increased (we still do not care about the equilibrium constraint), see Figs. 5-7. The transformation is done in two steps. First the points on the negative side of the halfspace are jointly folded into the positive side (Fig. 6 and Obs. 6), then all points are jointly collapsed (like an umbrella) into the flat halfspace (Fig. 7 and Obs. 7). Finally, in Obs. 8, we concat both transformations to one single transformation and exhibit some convenient properties. The algebraic proofs may be safely skipped by readers used to geometric reasoning.

Observation 6 (Folding along a subspace into a halfspace) Let $v_{i} \in \mathbb{R}^{n}$ for $i \in$ $K \subseteq N$, let $\mathcal{L} \subset \mathbb{R}^{n}$ be a subspace, and $b, h \in \mathcal{L}^{\perp}$ with $\|b\|=\|h\|=1, h^{T} b=0$ and $h^{T} v_{i}=0$ for $i \in K$. Define continuous maps $\varphi_{i}:[0,1] \rightarrow \mathbb{R}^{n}$ for $i \in K$ by

$$
\varphi_{i}(t)= \begin{cases}v_{i}-\left(v_{i}^{T} b\right) b+\left(v_{i}^{T} b\right)[b \cos t \pi+h \sin t \pi] & \text { if } v_{i}^{T} b<0 \\ v_{i} & \text { if } v_{i}^{T} b \geq 0 .\end{cases}
$$

Then $\varphi_{i}(0)=v_{i}, \varphi_{i}(1) \in\left(\operatorname{span}\left\{b, v_{i}: i \in K\right\}\right) \cap\left\{x \in \mathbb{R}^{n}: b^{T} x \geq 0\right\}$ and for all $t \in[0,1]$ it holds that $\left\|\varphi_{i}(t)-\varphi_{j}(t)\right\| \leq\left\|v_{i}-v_{j}\right\|$ for $i, j \in K$ and $\left\|\varphi_{i}(t)-v\right\|=\left\|v_{i}-v\right\|$ for $v \in \mathcal{L}$. In particular, $\sum_{i \in K}\left\|\varphi_{i}(t)\right\|^{2}$ is constant over all $t \in[0,1]$.

Proof. Let $\mathcal{U}=\left\{x \in \mathbb{R}^{n}: b^{T} x=0, h^{T} x=0\right\} \supseteq \mathcal{L}$, then $p_{\mathcal{U}}\left(v_{i}\right)=v_{i}-\left(v_{i}^{T} b\right) b$ for $i \in K$, because $h^{T} v_{i}=0$. Therefore $p_{\mathcal{U}}\left(v_{i}\right)=p_{\mathcal{U}}\left(\varphi_{i}(t)\right)$ and $\left\|\varphi_{i}(t)-v\right\|^{2}=\left\|\varphi_{i}(t)-p_{\mathcal{U}}\left(v_{i}\right)\right\|^{2}+$ 


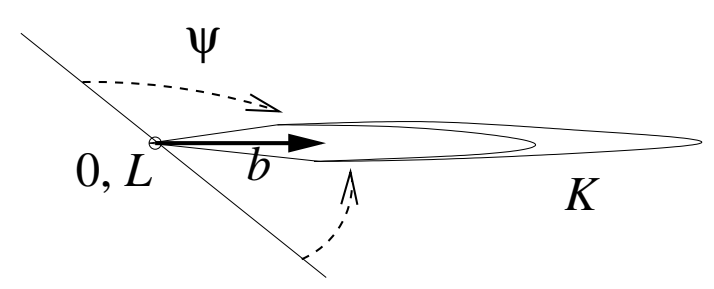

Figure 7: $\psi$ collapses $K$ into the flat halfspace spanned by $\mathcal{L}$ and direction $b$ (Obs. 7).

$\left\|p_{\mathcal{U}}\left(v_{i}\right)-v\right\|^{2}=\left|b^{T} v_{i}\right|^{2}+\left\|p_{\mathcal{U}}\left(v_{i}\right)-v\right\|^{2}$ for all $v \in \mathcal{U}$ (in particular for $v=0$ ), $t \in[0,1]$, $i \in K$. If, for $i, j \in K, v_{i}^{T} b \geq 0$ and $v_{j}^{T} b \geq 0$ then $\left\|\varphi_{i}(t)-\varphi_{j}(t)\right\|=\left\|v_{i}-v_{j}\right\|$ by definition; if $v_{i}^{T} b<0$ and $v_{j}^{T} b<0$ then $\left\|\varphi_{i}(t)-\varphi_{j}(t)\right\|^{2}=\left\|p_{\mathcal{U}}\left(v_{i}\right)-p_{\mathcal{U}}\left(v_{j}\right)\right\|^{2}+\left|v_{i}^{T} b-v_{j}^{T} b\right|^{2}=\left\|v_{i}-v_{j}\right\|^{2}$; if, w.l.o.g., $v_{i}^{T} b<0$ and $v_{j}^{T} b \geq 0$ use the point $v_{i j}=\left[v_{i}, v_{j}\right] \cap\left\{x \in \mathbb{R}^{n}: b^{T} x=0\right\} \in \mathcal{U}$ for $\left\|v_{i}-v_{j}\right\|=\left\|v_{i}-v_{i j}\right\|+\left\|v_{i j}-v_{j}\right\|=\left\|\varphi_{i}(t)-v_{i j}\right\|+\left\|v_{i j}-\varphi_{j}(t)\right\| \geq\left\|\varphi_{i}(t)-\varphi_{j}(t)\right\|$ by the triangle inequality.

Observation 7 (Collapsing a halfspace around a subspace into a flat halfspace) Let $\mathcal{L} \subset \mathbb{R}^{n}$ be a subspace, $b \in \mathcal{L}^{\perp}$ with $\|b\|=1$ and $v_{i} \in\left\{x \in \mathbb{R}^{n}: b^{T} x \geq 0\right\}$ for $i \in K \subseteq N$. For $i \in K$, let $\beta_{i}=\left\|p_{\mathcal{L}^{\perp}}\left(v_{i}\right)\right\|$ and $0 \leq \gamma_{i} \leq \frac{\pi}{2}, g_{i} \in \mathcal{L}^{\perp}, g_{i}^{T} b=0,\left\|g_{i}\right\|=1$ so that $p_{\mathcal{L}^{\perp}}\left(v_{i}\right)=\beta_{i}\left(g_{i} \cos \gamma_{i}+b \sin \gamma_{i}\right)$. Define continuous maps $\psi_{i}:[0,1] \rightarrow \mathbb{R}^{n}$ for $i \in K$ by

$$
\psi_{i}(t)=p_{\mathcal{L}}\left(v_{i}\right)+\beta_{i}\left[g_{i} \cos \left(\gamma_{i}+t\left[\frac{\pi}{2}-\gamma_{i}\right]\right)+b \sin \left(\gamma_{i}+t\left[\frac{\pi}{2}-\gamma_{i}\right]\right)\right] .
$$

Then $\psi_{i}(0)=v_{i}, \psi_{i}(1) \in \mathcal{L}+\{\beta b: \beta \geq 0\}$ and for all $t \in[0,1]$ it holds that $\left\|\psi_{i}(t)-\psi_{j}(t)\right\| \leq$ $\left\|v_{i}-v_{j}\right\|$ for $i, j \in K$ and $\left\|\psi_{i}(t)-v\right\|=\left\|v_{i}-v\right\|$ for $v \in \mathcal{L}$. In particular, $\sum_{i \in K}\left\|\psi_{i}(t)\right\|^{2}$ is constant over all $t \in[0,1]$.

Proof. By $g_{i}, b \in \mathcal{L}^{\perp}$ we obtain $p_{\mathcal{L}}\left(v_{i}\right)=p_{\mathcal{L}}\left(\psi_{i}(t)\right)$ and $\left\|\psi_{i}(t)-v\right\|^{2}=\left\|\psi_{i}(t)-p_{\mathcal{L}}\left(v_{i}\right)\right\|^{2}+$ $\left\|p_{\mathcal{L}}\left(v_{i}\right)-v\right\|^{2}=\beta_{i}^{2}+\left\|p_{\mathcal{L}}\left(v_{i}\right)-v\right\|^{2}$ for all $v \in \mathcal{L}$ (in particular for $v=0$ ), $t \in[0,1], i \in K$. For proving $\left\|\psi_{i}(t)-\psi_{j}(t)\right\|^{2} \leq\left\|v_{i}-v_{j}\right\|^{2}$ for $i, j \in K$ we show $\psi_{i}(t)^{T} \psi_{j}(t) \geq v_{i}^{T} v_{j}$ or $f(t)=\left(g_{i}^{T} g_{j}\right)\left[\cos \left(\gamma_{i}+t\left[\frac{\pi}{2}-\gamma_{i}\right]\right) \cos \left(\gamma_{j}+t\left[\frac{\pi}{2}-\gamma_{j}\right]\right)\right]+\sin \left(\gamma_{i}+t\left[\frac{\pi}{2}-\gamma_{i}\right]\right) \sin \left(\gamma_{j}+t\left[\frac{\pi}{2}-\gamma_{j}\right]\right) \geq$ $\left(g_{i}^{T} g_{j}\right)\left[\cos \gamma_{i} \cos \gamma_{j}\right]+\sin \gamma_{i} \sin \gamma_{j}=f(0)$. In the case $g_{i}^{T} g_{j}<0$ the inequality holds for $t \in[0,1]$ because both cosine terms in $f(t)$ are non increasing and the sine terms are non decreasing. In the remaining case we use the angle addition formulas to find

$$
f(t)=g_{i}^{T} g_{j} \cos \left((1-t)\left[\gamma_{i}-\gamma_{j}\right]\right)+\left(1-g_{i}^{T} g_{j}\right) \sin \left(\gamma_{i}+t\left[\frac{\pi}{2}-\gamma_{i}\right]\right) \sin \left(\gamma_{j}+t\left[\frac{\pi}{2}-\gamma_{j}\right]\right) .
$$

Then $f(t) \geq f(0)$ holds for $t \in[0,1]$ because $0 \leq g_{i}^{T} g_{j} \leq 1$ and the cosine and sine terms are non decreasing. 
Observation 8 (Concatenation of Folding and Collapsing) Let $v_{i} \in \mathbb{R}^{n}$ for $i \in K \subseteq$ $N$, let $\mathcal{L} \subset \mathbb{R}^{n}$ be a subspace, and $b, h \in \mathcal{L}^{\perp}$ with $\|b\|=\|h\|=1, h^{T} b=0$ and $h^{T} v_{i}=0$ for $i \in K$. Define continuous maps $v_{i}:[0,1] \rightarrow \mathbb{R}^{n}$ for $i \in K$ by

$$
v_{i}(t)= \begin{cases}\varphi_{i}(2 t) & \text { for } t \in\left[0, \frac{1}{2}\right] \\ \psi_{i}\left(2\left[t-\frac{1}{2}\right]\right) & \text { for } t \in\left(\frac{1}{2}, 1\right]\end{cases}
$$

where $\varphi_{i}$ is as given in Obs. 6 and $\psi_{i}$ of Obs. 7 starts with the embedding $v_{i}^{\prime}=\varphi_{i}(1)$, $i \in K$. Then $v_{i}(0)=v_{i}, v_{i}(1) \in \mathcal{L}+\{\beta b: \beta \geq 0\}$ and for all $t \in[0,1]$ it holds that $\left\|v_{i}(t)-v_{j}(t)\right\| \leq\left\|v_{i}-v_{j}\right\|$ for $i, j \in K$ and $\left\|v_{i}(t)-v\right\|=\left\|v_{i}-v\right\|$ for $v \in \mathcal{L}$. In particular, $\sum_{i \in K}\left\|v_{i}(t)\right\|^{2}$ is constant over all $t \in[0,1]$.

Furthermore, for $\bar{v}(t)=\sum_{i \in K} v_{i}(t)$ and $\mathcal{B}=\mathcal{L}+\operatorname{span}\{b\}$ the function $\lambda(t)=\bar{v}(t)^{T} b$ is nondecreasing and continuous with $p_{\mathcal{B}}(\bar{v}(t))=p_{\mathcal{L}}(\bar{v}(0))+\lambda(t) b$. In particular, $\bar{v}(1)=$ $p_{\mathcal{L}}(\bar{v}(0))+\lambda(1) b$ and

$$
\lambda(0) \leq\left\|p_{\mathcal{L}^{\perp}}(\bar{v}(t))\right\| \leq\left\|p_{\mathcal{L}^{\perp}}(\bar{v}(1))\right\|=\lambda(1)=\sum_{i \in K}\left\|p_{\mathcal{L}^{\perp}}\left(v_{i}\right)\right\| \quad \forall t \in[0,1],
$$

so $\lambda(1)$ is independent of the choice of $b$.

Proof. The stated properties of $v_{i}(t), i \in K$, follow directly from Obs. 6 and 7. Turning to $\bar{v}(t)$, observe that $p_{\mathcal{L}}\left(v_{i}\right)=p_{\mathcal{L}}\left(v_{i}(t)\right)$ and $\frac{d\left(b^{T} v_{i}(t)\right)}{d t} \geq 0$ for all $t \in[0,1], i \in K$, so for each $i \in K, \lambda_{i}(t)=b^{T} v_{i}(t)$ is a nondecreasing function with $p_{\mathcal{B}}\left(v_{i}(t)\right)=p_{\mathcal{L}}\left(v_{i}\right)+\lambda_{i}(t) b$. Furthermore, $\lambda_{i}(1)=\left\|p_{\mathcal{L}^{\perp}}\left(v_{i}\right)\right\|$ because $\left\|v_{i}\right\|=\left\|v_{i}(1)\right\|$ and this value is independent of the choice of $b$. Therefore this also holds for $\lambda(\cdot)=\sum_{i \in K} \lambda_{i}(\cdot)$.

We need yet another operation similar in style to Obs. 7 , but this time we squeeze vectors in a flat halfspace relative to the invariant subspace into a halfspace of its boundary (comparable to closing a fan, see Fig. 8).

\section{Observation 9 (Squeezing a halfspace into a halfspace of its boundary)}

Let $\mathcal{L} \subset \mathbb{R}^{n}$ be a subspace, $b, g \in \mathcal{L}^{\perp}$ with $\|b\|=\|g\|=1, b^{T} g=0$ and $v_{i} \in\{x \in$ $\left.\mathcal{L}+\operatorname{span}\{b, g\}: b^{T} x \geq 0\right\}$ for $i \in K \subseteq N$. For $i \in K$, let $\beta_{i}=\left\|p_{\mathcal{L}^{\perp}}\left(v_{i}\right)\right\|$ and $0 \leq \gamma_{i} \leq \pi$ so that $p_{\mathcal{L}^{\perp}}\left(v_{i}\right)=\beta_{i}\left(g \cos \gamma_{i}+b \sin \gamma_{i}\right)$. Define continuous maps $\theta_{i}:[0,1] \rightarrow \mathbb{R}^{n}$ for $i \in K$,

$$
\theta_{i}(t)=p_{\mathcal{L}}\left(v_{i}\right)+\beta_{i}\left[g \cos \left(\gamma_{i}-t \gamma_{i}\right)+b \sin \left(\gamma_{i}-t \gamma_{i}\right)\right] .
$$

Then $\theta_{i}(0)=v_{i}, \theta_{i}(1) \in \mathcal{L}+\{\beta g: \beta \geq 0\}$ and for all $t \in[0,1]$ it holds that $\left\|\theta_{i}(t)-\theta_{j}(t)\right\| \leq$ $\left\|v_{i}-v_{j}\right\|$ for $i, j \in K,\left\|\theta_{i}(t)-v\right\|=\left\|v_{i}-v\right\|$ for $v \in \mathcal{L}$ and $\left\|\theta_{i}(t)-v\right\| \leq\left\|v_{i}-v\right\|$ for $v \in \mathcal{L}+\{\beta g: \beta \geq 0\}$. In particular, $\sum_{i \in K}\left\|\theta_{i}(t)\right\|^{2}$ is constant over all $t \in[0,1]$ and $\sum_{i \in K} \theta_{i}(t)^{T} g$ is nondecreasing.

Proof. By $g, b \in \mathcal{L}^{\perp}$ we obtain $p_{\mathcal{L}}\left(v_{i}\right)=p_{\mathcal{L}}\left(\theta_{i}(t)\right)$ and $\left\|\theta_{i}(t)-v\right\|^{2}=\left\|\theta_{i}(t)-p_{\mathcal{L}}\left(v_{i}\right)\right\|^{2}+$ $\left\|p_{\mathcal{L}}\left(v_{i}\right)-v\right\|^{2}=\beta_{i}^{2}+\left\|p_{\mathcal{L}}\left(v_{i}\right)-v\right\|^{2}$ for all $v \in \mathcal{L}$ (in particular for $v=0$ ), $t \in[0,1]$, $i \in K$. For proving $\left\|\theta_{i}(t)-\theta_{j}(t)\right\|^{2} \leq\left\|v_{i}-v_{j}\right\|^{2}$ for $i, j \in K$ we show $\theta_{i}(t)^{T} \theta_{j}(t) \geq v_{i}^{T} v_{j}$ or $\cos \left(\gamma_{i}-t \gamma_{i}\right) \cos \left(\gamma_{j}-t \gamma_{j}\right)+\sin \left(\gamma_{i}-t \gamma_{i}\right) \sin \left(\gamma_{j}-t \gamma_{j}\right) \geq \cos \gamma_{i} \cos \gamma_{j}+\sin \gamma_{i} \sin \gamma_{j}$. By the angle 


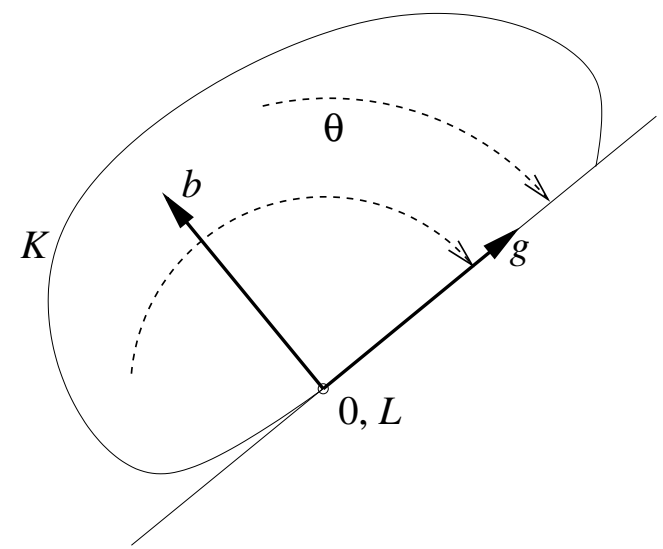

Figure 8: $\theta$ squeezes $K$ spanned by $\mathcal{L}+\operatorname{span}\{g\}$ and nonnegative $b$ into the boundary halfspace spanned by $\mathcal{L}$ and nonnegative $g$. (Obs. 9).

addition formulas and since the cosine is an even function we may rewrite the inequality to $\cos \left((1-t)\left|\gamma_{i}-\gamma_{j}\right|\right) \geq \cos \left(\left|\gamma_{i}-\gamma_{j}\right|\right)$. This holds for $t \in[0,1]$ because $0 \leq\left|\gamma_{i}-\gamma_{j}\right| \leq \pi$ and the left hand cosine term is non decreasing. Finally, this also holds true for arbitrary $i \in K$ and all $v \in \mathcal{L}+\{\beta g: \beta \geq 0\}$ as can be observed by setting, e.g., $\gamma_{j}=0$ above.

The last observation of this section will be helpful in establishing the equilibrium constraint.

Observation 10 Given scalars $\beta_{j}>0, j \in M\{1, \ldots, m\}, m \geq 2$, so that, for each $\hat{\jmath} \in M$, $\sum_{j \in M \backslash\{\hat{\jmath}\}} \beta_{j} \geq \beta_{\hat{\jmath}}$ then there exist vectors $d_{1}, d_{2}, d_{3} \in \mathbb{R}^{2}$ with $\left\|d_{1}\right\|=\left\|d_{2}\right\|=\left\|d_{3}\right\|=1$ and an assignment $\kappa: M \rightarrow\{1,2,3\}$ so that $\sum_{j \in M} \beta_{j} d_{\kappa(j)}=0$. This also holds, if in addition $|\{j \in M: \kappa(j)=1\}|=1$ is required.

Proof. If $|M|=2$ then $\beta_{1}=\beta_{2}$ and the claim holds for $d_{1}=-d_{2}$ and $\kappa$ correspondingly. Otherwise let $\hat{\jmath} \in M$ be the smallest number so that $\sum_{j=1}^{\hat{\jmath}-1} \beta_{j}<\frac{1}{2} \sum_{j \in M} \beta_{j} \leq \sum_{j=1}^{\hat{\jmath}} \beta_{j}$, set $\kappa(\hat{\jmath})=1, \kappa(j)=2$ for $\hat{\jmath}>j \in M$ and $\kappa(j)=3$ for $\hat{\jmath}<j \in M$. Let $\bar{\beta}_{h}=\sum_{j \in M, \kappa(j)=h} \beta_{j}$, $h \in\{1,2,3\}$ and assume w.l.o.g. (by changing the numbering, if required) that the relations $\bar{\beta}_{1} \leq \bar{\beta}_{2} \leq \bar{\beta}_{3}$ and $\bar{\beta}_{1}+\bar{\beta}_{2} \geq \bar{\beta}_{3}$ hold. Set $d_{1}(\alpha)=(\cos \alpha,-\sin \alpha)^{T}$ for $0 \leq \alpha \leq \pi$, $d_{2}(\alpha)=(\cos \gamma(\alpha), \sin \gamma(\alpha))^{T}$ for $\beta_{2} \sin \gamma(\alpha)=\beta_{1} \sin \alpha$ and $d_{3}=(-1,0)^{T}$. Then $b(\alpha)=$ $\bar{\beta}_{1} d_{1}(\alpha)+\bar{\beta}_{2} d_{2}(\alpha)+\bar{\beta}_{3} d_{3}$ satisfies $[b(\alpha)]_{2}=0$ for all $0 \leq \alpha \leq \pi,[b(0)]_{1} \geq 0$ and $[b(\pi)]_{1} \leq 0$, so by continuity of $b(\alpha)$ there is a $\bar{\alpha} \in[0, \pi]$ with $b(\bar{\alpha})=0$.

\section{Properties of Optimal Embeddings of Graphs}

We first note, that a feasible embedding cannot be full dimensional.

Observation 11 The dimension of any feasible embedding is at most $n-1$, i.e., let $v_{i} \in \mathbb{R}^{n}$ satisfy the constraints of (4) then there is a vector $h \in \mathbb{R}^{n},\|h\|=1$, with $h^{T} v_{i}=0$ for all $i \in N$. 


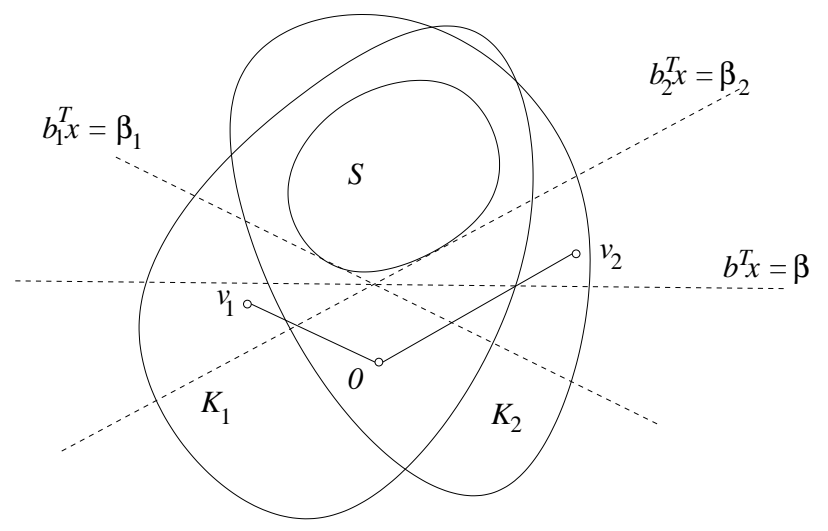

Figure 9: Initial setting in case 1 of the separator-shadow proof.

Proof. The $n$ vectors $v_{i}$ satisfy $\sum_{i \in N} v_{i}=0$, so they are linearly dependent and therefore $\operatorname{dim}\left(\operatorname{span}\left\{v_{1}, \ldots, v_{n}\right\}\right) \leq n-1$.

Our main result on the structure of optimal embeddings is the following.

Theorem 12 (Separator-Shadow) Let $v_{i} \in \mathbb{R}^{n}$ for $i \in N$ be an optimal solution of (4) for a connected graph $G=(N, E)$ and let $K_{1} \cup S \cup K_{2}$ be a partition of $N$ with no node in $K_{1}$ adjacent to a node in $K_{2}$. Then, for at least one $j \in\{1,2\}$, for every $i \in K_{j}$ the straight line segment $\left[0, v_{i}\right]$ intersects the convex hull of the points in $S$, i.e., $\forall i \in K_{j}$ : $\left[0, v_{i}\right] \cap \operatorname{conv}\left\{v_{s}: s \in S\right\} \neq \emptyset$.

If $K_{1}$ and $K_{2}$ are both nonempty then $S$ is a separator in $G$. Note that the theorem holds trivially if the origin is contained in the convex hull of the points in $S$ or if one of the sets $S, K_{1}, K_{2}$ is empty (if $S=\emptyset$ then one of $K_{1}$ and $K_{2}$ must also be empty by the connectedness of $G$ ).

Proof. Let $h \in \mathbb{R}^{n}$ with $\|h\|=1$ satisfy $h^{T} v_{i}=0$ for all $i \in N$ as in Obs. 11 and let $\mathcal{S}=\operatorname{conv}\left\{v_{s}: s \in S\right\}$. Assume, for contradiction, that the theorem is not true. Then, w.l.o.g., there are points $v_{1}, v_{2}$ with $1 \in K_{1}, 2 \in K_{2}$ and $\left[0, v_{1}\right] \cap \mathcal{S}=\left[0, v_{2}\right] \cap \mathcal{S}=\emptyset$ (the set $S$ cannot be empty as $K_{1}$ and $K_{2}$ are not). By convex separation there exist, for $j \in\{1,2\}$, separating hyperplanes $b_{j} \in \mathbb{R}^{n}$ with $h^{T} b_{j}=0$ and $\beta_{j}>0$ within the subspace spanned by the $v_{i}$ so that $b_{j}^{T} x \geq \beta_{j}$ for all $x \in \mathcal{S}$ and $b_{j}^{T} x<\beta_{j}$ for all $x \in\left[0, v_{j}\right]$.

Note, that there cannot exist $\alpha \in[0,1], b=\alpha b_{1}+(1-\alpha) b_{2}, \beta=\alpha \beta_{1}+(1-\alpha) \beta_{2}$ so that $\left\langle b, v_{i}\right\rangle \geq \beta$ for all $i \in K_{1} \cup K_{2}$, because the hyperplane $\langle b, x\rangle=\beta / 2>0$ would separate 0 strictly from $\operatorname{conv}\left\{v_{i}: i \in N\right\}$, but by feasibility of the $v_{i}$ the origin is a convex combination of the $v_{i}$ as $\frac{1}{n} \sum_{i \in N} v_{i}=0$.

Hence, there must exist an $\alpha \in[0,1]$ so that for $b=\alpha b_{1}+(1-\alpha) b_{2}, \beta=\alpha \beta_{1}+(1-\alpha) \beta_{2}$ the open halfspace $\langle b, x\rangle<\beta$ contains points of both $K_{1}$ and $K_{2}$ (illustrated in Fig. 9).

Note, that $b^{T} h=0$ holds and by scaling $b$ and $\beta$ we may assume w.l.o.g. $\|b\|=1$. Let, for $j \in\{1,2\}, M_{j}=\left\{i \in K_{j}:\left\langle b, v_{i}\right\rangle<\beta\right\}, m_{j}=\left|M_{j}\right|>0$, and $\bar{v}_{j}=\frac{1}{m_{j}} \sum_{i \in M_{j}} v_{i}$. Next, consider rotating independently for each $j$ the points in $M_{j}$ around the affine subspace 


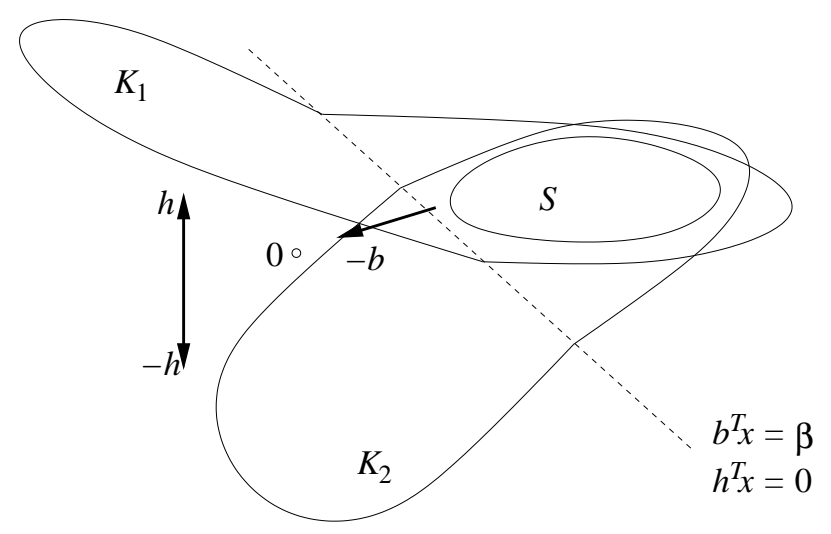

Figure 10: Improving movement in Case 1 of the separator-shadow proof.

$L=\left\{x \in \mathbb{R}^{n}:\langle h, x\rangle=0,\langle b, x\rangle=\beta\right\}$. Because the points in $M_{1}$ and $M_{2}$ are not adjacent and distances to the remaining points are not increased, the edge constraints in (4) remain satisfied. We show, that rotating the points in $M_{1}$ in direction $h$ and the points in $M_{2}$ against direction $h$ by sufficiently small angels $\gamma_{1}$ and $\gamma_{2}$ improves the solution (see Fig. 10). As in the proof of Obs. 5 denote, for $j \in\{1,2\}$, by $r_{j}=\beta-b^{T} \bar{v}_{j}>0$ the radius and by $d_{j}=r_{j}\left[\left(\sin \gamma_{j}\right) h+\left(1-\cos \gamma_{j}\right) b\right]$ the displacement of $\bar{v}_{j}$ yielding the improvement $2 m_{j} r_{j}\left(1-\cos \gamma_{j}\right) \beta$. Rotation $j$ adds $m_{j} d_{j}$ to the barycenter of all points and has to be compensated in order to maintain feasibility. Shifts of the barycenter in the direction of $h$ can be avoided by requiring $m_{1} d_{1}^{T} h=-m_{2} d_{2}^{T} h$, i.e., given $\gamma_{1}$ choose $\gamma_{2}$ so that $m_{1} r_{1} \sin \gamma_{1}=-m_{2} r_{2} \sin \gamma_{2}$. After carrying out these rotations it therefore remains to shift all points by $d=-\left(m_{1} d_{1}^{T} b+m_{2} d_{2}^{T} b\right) b / n=-\left[m_{1} r_{1}\left(1-\cos \gamma_{1}\right)+m_{2} r_{2}\left(1-\cos \gamma_{2}\right)\right] b / n$ for feasibility in (4). Using Obs. 4, the total objective improvement is

$$
\begin{aligned}
& \sum_{j \in\{1,2\}} 2 m_{j} r_{j}\left(1-\cos \gamma_{j}\right) \beta-n\langle d, d\rangle= \\
& =\sum_{j \in\{1,2\}} 2 m_{j} r_{j}\left(1-\cos \gamma_{j}\right) \beta-\frac{1}{n}\left[m_{1} r_{1}\left(1-\cos \gamma_{1}\right)+m_{2} r_{2}\left(1-\cos \gamma_{2}\right)\right]^{2} .
\end{aligned}
$$

This is positive for $\gamma_{1}$ and $\gamma_{2}\left(\gamma_{1}\right)$ close enough to zero, yielding a contradiction to the optimality of the embedding.

The theorem allows to derive several further structural properties.

Corollary 13 Let $v_{i} \in \mathbb{R}^{n}$ for $i \in N$ describe an optimal solution of (4) for a connected graph $G=(N, E)$ and let $S$ be a separator in $G$, i.e., there is a $K \subseteq N \backslash S$ and no node in $K$ is adjacent to a node in $N \backslash(S \cup K)$. Then the convex hull of the points in $S$ intersects the straight line segment between the barycenter $\bar{v}$ of the points in $K$ and the barycenter $\underline{v}$ of the points in $N \backslash K$.

Proof. Let $\mathcal{S}=\operatorname{conv}\left\{v_{s}: s \in S\right\}, \bar{v}=\frac{1}{|K|} \sum_{i \in K} v_{i}$, and $\underline{v}=\frac{1}{|N \backslash K|} \sum_{i \in N \backslash K} v_{i}=-\frac{|K|}{|N \backslash K|} \bar{v}$ (by $\sum_{i \in N} v_{i}=0$ ), so $0 \in[\bar{v}, \underline{v}]$ and it suffices to prove that $[\bar{v}, 0] \cap \mathcal{S} \neq \emptyset$ or $[\underline{v}, 0] \cap \mathcal{S} \neq \emptyset$. 
Setting $K_{1}=K, K_{2}=N \backslash(S \cup K)$, Th. 12 implies that for at least one of $j \in\{1,2\}$ we have $[v, 0] \cap \mathcal{S} \neq \emptyset$ for all $v \in\left\{v_{i}: i \in K_{j}\right\}$, and therefore also for all $v \in \operatorname{conv}\left\{v_{i}: i \in K_{j}\right\}$ and for all $v \in \operatorname{conv}\left\{v_{i}: i \in K_{j} \cup S\right\}$. If $j=1$ then $\bar{v} \in \operatorname{conv}\left\{v_{i}: i \in K_{1}\right\}$, if $j=2$ then $\underline{v} \in \operatorname{conv}\left\{v_{i}: i \in K_{2} \cup S\right\}$, which proves the corollary.

Remark 14 The typical bisection procedure for Fiedler-vectors is to take an eigenvector $v$ of $\lambda_{2}(L)$ and partition the nodes according to $K_{1}=\left\{i \in N: v_{i}<0\right\}$ and $K_{2}=\{i \in$ $\left.N: v_{i}>0\right\}$. A first improvement suggested by the embedding is to use - instead of an arbitrary eigenvector to $\lambda_{2}(L)$ - an eigenvector to the maximum eigenvalue of an optimal solution $X$ of (3).

According to Cor. 13, the following heuristic might do an even better job in finding good separators or bisections into parts of size between one and two thirds of all nodes. Project the points onto a random hyperplane (like in the approach of Goemans and Williamson [11] and maybe biased by the eigenvalue/eigenvector structure of $X$ as suggested in [1]), yielding an assignment of the points to an interval on the real line. If the hyperplane happens to be close to perpendicular to the affine hull of a desired separator, then the points within the separator should be mapped close to each other and the majority of the remaining points should be mapped to the correct respective sides. So, when looking at the middle third of points within this interval and checking for good separators within a fixed number of adjacent points, chances should be reasonable to locate an acceptable candidate. In particular, if the embedding is low dimensional (see Th. 19 and Remark 20 below), relatively few random hyperplanes will suffice to obtain a suitable hyperplane with high probability. Whether this approach is indeed successful in practice has to be explored in future research.

Theorem 12 is not very descriptive, if the convex hull of the separator contains the origin. The following result improves on this and will help to establish a nontrivial bound on the dimension of an optimal embedding of minimal dimension.

Theorem 15 Let $v_{i} \in \mathbb{R}^{n}$ for $i \in N$ be an optimal solution of (4) for a connected graph $G=(N, E)$ and let $S \subset N$ with $0 \in \mathcal{S}=\operatorname{conv}\left\{v_{s}: s \in S\right\}$ be a separator in $G$ inducing a partition $\left(S, K_{1}, \ldots, K_{m}\right)$ of $N$ so that no node in $K_{j}$ is adjacent to a node in $K_{h}$ for $j \neq h, j, h \in M=\{1, \ldots, m\}$. Set $\mathcal{L}=\operatorname{span} \mathcal{S}$ and, for $j \in M, \beta_{j}=\sum_{i \in K_{j}}\left\|p_{\mathcal{L}^{\perp}}\left(v_{i}\right)\right\|$.

(i) If $\beta_{\hat{\jmath}}>\sum_{j \in M \backslash\{\hat{\jmath}\}} \beta_{j}$ for one $\hat{\jmath} \in M$ then there exist $h \in \mathcal{L}^{\perp}$ and an optimal embedding $v_{i}^{\prime} \in \mathbb{R}^{n}$ of (4) with $v_{i}^{\prime}=v_{i}$ for $i \in S, v_{i}^{\prime} \in \mathcal{L}+\operatorname{span}\left\{h, v_{i}: i \in K_{\hat{\jmath}}\right\}$ for $i \in K_{\hat{\jmath}}$ and $v_{i}^{\prime} \in \mathcal{L}+\left\{\beta \sum_{i \in K_{\hat{\jmath}}} v_{i}^{\prime}: \beta \geq 0\right\}$ for $i \in \bigcup_{j \in M \backslash\{\hat{\jmath}\}} K_{j}$. If, in addition, there exists $\bar{b} \in \operatorname{span}\left\{v_{i}: i \in K_{\hat{\jmath}}\right\},\|\bar{b}\|=1$ so that $\left\langle\bar{b}, v_{i}\right\rangle \geq 0$ for all $i \in K_{\hat{\jmath}}$, then such an embedding exists with $h=0$.

(ii) If $\beta_{\hat{\jmath}} \leq \sum_{j \in M \backslash\{\hat{\jmath}\}} \beta_{j}$ for all $\hat{\jmath} \in M$ then there exist vectors $d_{1}, d_{2}, d_{3} \in \mathcal{L}^{\perp},\left\|d_{1}\right\|=$ $\left\|d_{2}\right\|=\left\|d_{3}\right\|=1$ with dimspan $\left\{d_{1}, d_{2}, d_{3}\right\} \leq 2, b_{j} \in\left\{d_{1}, d_{2}, d_{3}\right\}, j \in M$, and an optimal embedding $v_{i}^{\prime} \in \mathbb{R}^{n}, i \in N$, of (4) with $v_{i}^{\prime}=v_{i}$ for $i \in S$ so that for each $j \in M$ we have $v_{i}^{\prime} \in \mathcal{L}+\left\{\beta b_{j}: \beta \geq 0\right\}$ for all $i \in K_{j}$. One may assume $b_{j}=d_{1}$ for at most one $j \in M$. 
(iii) If, in case (ii), the index $\hat{\jmath} \in M$ is the only $j \in M$ satisfying $b_{j}=d_{1}$ and at most $|S|-1$ nodes of $S$ are adjacent to nodes in $K_{\hat{\jmath}}$, then there is an optimal embedding of dimension at most $|S|$.

Proof. We start by eliminating some easy cases. If there is no $i \in N$ with $v_{i} \notin \mathcal{L}$ then $\beta_{j}=0$ for all $j \in M$, thus case (ii) holds and the dimension of the embedding is bounded by $|S|-1$ without making use of vectors $d_{1}, d_{2}, d_{3}$. So assume that there is an $h \in M$ with $v_{i} \notin \mathcal{L}$ for some $i \in K_{h}$. For $j \in M$ such that $v_{i} \in \mathcal{L}$ for all $i \in K_{j}$ the embedding will not be changed in the proof and we may add, for notational convenience, all such points jointly to $K_{h}$ and remove $j$ from $M$. If, after this preprocessing step, $|M| \leq 1$, then case (i) of the theorem holds for $v_{i}^{\prime}=v_{i}, i \in N$, and $h=0$. So assume, w.l.o.g., that $M=\{1, \ldots, m\}$ for some $m \geq 2$ and for all $j \in M$, the $K_{j}$ contain points outside $\mathcal{L}$. For convenience we set $H_{1}=K_{1} \cup S$ and $H_{j}=K_{j}$ for $j \in M \backslash\{1\}$.

For each $j \in M$ consider fixing, as a parameter to be specified later, a goal direction $b_{j} \in \mathcal{L}^{\perp}$ with $\left\|b_{j}\right\|=1$ and set $\mathcal{B}_{j}^{+}=\left\{\mathcal{L}+\beta b_{j}: \beta \geq 0\right\}$. For each $H_{j}, j \in M$, and $i \in H_{j}$ let $v_{i}(t), \bar{v}_{j}(t)=\sum_{i \in H_{j}} v_{i}(t), \lambda_{j}(t)$ denote the transformations/maps of Obs. 8 (set $K=H_{j}$, $b=b_{j}$, use the current $\mathcal{L}$ and choose any $h \in \mathcal{L}^{\perp}$ with $\|h\|=1$ and $b_{j}^{T} h=0$ as in Obs. 11 exploiting $|K|<|N|)$. The transformation maintains feasibility in the distance constraints but does not care about the equilibrium constraint.

By feasibility of the initial solution

$$
\sum_{j \in M} \sum_{i \in H_{j}} v_{i}=\sum_{j \in M} \bar{v}_{j}(0)=\sum_{j \in M} p_{\mathcal{L}}\left(\bar{v}_{j}(0)\right)+\sum_{j \in M} p_{\mathcal{L}^{\perp}}\left(\bar{v}_{j}(0)\right)=0,
$$

and therefore

$$
\sum_{j \in M} \sum_{i \in H_{j}} p_{\mathcal{L}}\left(v_{i}(0)\right)=\sum_{j \in M} \sum_{i \in H_{j}} p_{\mathcal{L}}\left(v_{i}\left(t_{j}\right)\right)=\sum_{j \in M} p_{\mathcal{L}}\left(\bar{v}_{j}\left(t_{j}\right)\right)=0 \quad \text { for } t_{j} \in[0,1], j \in M,
$$

independent of the choice of the $b_{j}, j \in M$. Therefore it remains to find $b_{j}$ and $t_{j}$ that achieve equilibrium in the space orthogonal to $\mathcal{L}$. For $j \in M, \beta_{j}=\lambda_{j}(1)=\sum_{i \in H_{j}}\left\|p_{\mathcal{L}^{\perp}}\left(v_{i}\right)\right\|$ (see Obs. $8 ; \beta_{j}>0$ by assumption) and assume, w.l.o.g., that $\beta_{1} \geq \beta_{j}$ for all $j \in M$. Now consider the following cases.

Case (i): $\beta_{1}>\sum_{j=2}^{m} \beta_{j}$. If there exists $\bar{b} \in \operatorname{span}\left\{v_{i}: i \in H_{1}\right\},\|\bar{b}\|=1$, satisfying $\bar{b}^{T} v_{i} \geq 0$ for $i \in H_{1}$, set $b_{1}=\bar{b}$, otherwise set $b_{1}=\frac{v_{i}-p_{\mathcal{L}}\left(v_{i}\right)}{\left\|v_{i}-p_{\mathcal{L}}\left(v_{i}\right)\right\|}$ for some $i \in H_{1}$ with $v_{i} \notin \mathcal{L}$. By Obs. 8, $\left\|p_{\mathcal{L}^{\perp}}\left(\bar{v}_{j}(t)\right)\right\| \leq \beta_{j}$ for $j \in M, t \in[0,1]$, so $(5)$ implies $\left\|p_{\mathcal{L}^{\perp}}\left(\bar{v}_{1}(0)\right)\right\| \leq$ $\sum_{j=2}^{m} \beta_{j}$. Considered as a function of $t \in[0,1]$, the norm $\left\|p_{\mathcal{L}^{\perp}}\left(\bar{v}_{1}(t)\right)\right\|$ is continuous with $\left\|p_{\mathcal{L}^{\perp}}\left(\bar{v}_{1}(1)\right)\right\|=\beta_{1}$, so there must be a $\bar{t} \in[0,1]$ with $\left\|p_{\mathcal{L}^{\perp}}\left(\bar{v}_{1}(\bar{t})\right)\right\|=\sum_{j=2}^{m} \beta_{j}$. Now set $b_{j}=-\frac{\bar{v}_{1}(\bar{t})-p_{\mathcal{L}}\left(\bar{v}_{1}(\bar{t})\right)}{\left.\| \bar{v}_{1}(\bar{t})-p_{\mathcal{L}} \bar{v}_{1}(\bar{t})\right) \|}$. Then the embedding $v_{i}^{\prime \prime}=v_{i}(\bar{t})$ for $i \in H_{1}$ and $v_{i}^{\prime \prime}=v_{i}(1)$ for $i \in H_{j}$ with $j \in M \backslash\{1\}$ is feasible, has optimal value and satisfies the requirements of the theorem, where $h$ is the additional direction needed if $\bar{t}<\frac{1}{2}$ (the folding part $\varphi$ of Obs. 6 within $v(\cdot)$ of Obs. 8). If $\bar{b}$ exists, no folding is needed and we can always find a $\bar{t} \geq \frac{1}{2}$.

Case (ii): $\beta_{1} \leq \sum_{j=2}^{m} \beta_{j}$. By Obs. 10 we can find three vectors $d_{1}, d_{2}, d_{3} \in \mathbb{R}^{n},\left\|d_{1}\right\|=$ $\left\|d_{2}\right\|=\left\|d_{3}\right\|=1$, in a plane orthogonal to $\mathcal{L}$ and a corresponding assignment $\kappa: M \rightarrow$ 

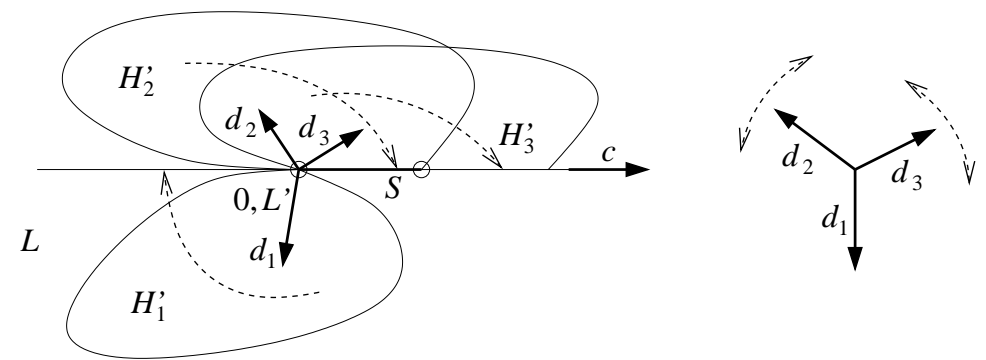

Figure 11: Transformation in the proof of Th. 15 (iii).

$\{1,2,3\}$ with $|\{j \in M: \kappa(j)=1\}|=1$ so that $\sum_{j \in M} \beta_{j} d_{\kappa(j)}=0$. Thus, setting $b_{j}=d_{\kappa(j)}$ we obtain a feasible embedding $v_{i}^{\prime}=v_{i}(1)$ for $i \in H_{j}, j \in M$ with the same (and therefore optimal) objective value.

Case (iii): Within this setting of Case (ii) let $\hat{\jmath} \in M$ be the only index with $b_{\jmath}=d_{1}$ and assume that, with respect to $S$, the vertices of $K_{\hat{\jmath}}$ are adjacent only to a subset $S^{\prime} \subset S$ with $\left|S^{\prime}\right|<|S|$. For ease of notation, let $H_{h}^{\prime}=\bigcup_{j \in M: \kappa(j)=h} K_{j}$ for $h \in\{1,2\}$ and $H_{3}^{\prime}=\bigcup_{j \in M: \kappa(j)=3} K_{j} \cup S$. If $\operatorname{dim} \mathcal{L}<|S|-1$ the claim holds by Case (ii), so assume, the $v_{i}^{\prime}, i \in S$, span an $|S|-1$ dimensional simplex. If $0 \notin \operatorname{conv}\left\{v_{s}: s \in S^{\prime}\right\}$, then the separator-shadow Th. 12 asserts that $v_{i}^{\prime} \in \mathcal{L}$ for $i \in H_{1}^{\prime}$, and this case was dealt with in the beginning of the proof. Thus, it suffices to consider the case $0 \in \operatorname{conv}\left\{v_{s}: s \in S^{\prime}\right\}$.

We will transform the embedding $v_{i}^{\prime}$ and reduce its dimension by one. Let $\bar{s} \in S \backslash S^{\prime}$ and set $\mathcal{L}^{\prime}=\operatorname{span} \operatorname{conv}\left\{v_{s}: \bar{s} \neq s \in S\right\}, c=\frac{v_{s}^{\prime}-p_{\mathcal{L}^{\prime}}}{\left\|v_{s}^{\prime}-p_{\mathcal{L}^{\prime}}\right\|}$. Observe (see Fig. 11), that $d_{h}^{T} c=0$ for $h \in\{1,2,3\}, v_{s}^{\prime} \in \mathcal{L}^{\prime}$ for $s \in S^{\prime}, v_{s}^{\prime} \in \mathcal{L}^{\prime}+\{\beta c: \beta \geq 0\}$ for $s \in S$, and $v_{i}^{\prime} \in \mathcal{L}^{\prime}+\operatorname{span}\{c\}+\left\{\beta d_{h}: \beta \geq 0\right\}$ for $h \in\{1,2,3\}, i \in H_{h}^{\prime}$. Next employ Obs. 9 and define for $h \in\{1,2,3\}$ the transformations $v_{i}^{\prime}(t)=\theta_{i}^{h}(t), i \in H_{h}^{\prime}$, setting in Obs. $9, \mathcal{L}=\mathcal{L}^{\prime}$, $b=d_{h}, g=-c$ for $h=1, g=c$ for $h \in\{2,3\}, K=H_{h}^{\prime}$ and $\theta_{i}^{h}(0)=v_{i}^{\prime}$. These squeeze $H_{1}^{\prime}$ into $\mathcal{L}^{\prime}+\{\beta c: \beta \leq 0\}$ and $H_{2}^{\prime}, H_{3}^{\prime}$ into $\mathcal{L}^{\prime}+\{\beta c: \beta \geq 0\}$ in a fan-like manner within the corresponding flat halfspaces spanned by $d_{1}, d_{2}, d_{3}$ respectively. Observe, that $v_{i}^{\prime}(t)=v_{i}^{\prime}$ for $i \in S, t \in[0,1]$ and that for $h \in\{1,2,3\}, i, j \in H_{h}^{\prime} \cup S$ the distances $\left\|v_{i}^{\prime}(t)-v_{j}^{\prime}(t)\right\|$ are nonincreasing for $t \in[0,1]$. Furthermore the value of the cost function does not change. The equilibrium constraint is influenced by the movement of the points $\bar{v}_{h}^{\prime}(t)=$ $\sum_{i \in H_{h}^{\prime}} v_{i}^{\prime}(t)$ for $t \in[0,1], h \in\{1,2,3\}$. Their projections onto $\mathcal{L}^{\prime}$ are invariant, therefore $\sum_{h \in\{1,2,3\}} p_{\mathcal{L}^{\prime}}\left(v_{h}^{\prime}(t)\right)=0$ throughout. The only potential infeasibilities caused by squeezing subgraph $h \in\{1,2,3\}$ are in directions $d_{h}$ (with $\bar{v}_{h}^{\prime}(t)^{T} d_{h} \geq 0$ throughout) and $c$ (with $\bar{v}_{h}^{\prime}(t)^{T} c$ nonincreasing for $h=1$ and nondecreasing for $\left.h \in\{2,3\}\right)$. Set $\bar{\beta}_{h}(t)=\left\|p_{\mathcal{L}^{\perp}}(\bar{v}(t))\right\|$. Suppose $t^{h} \in[0,1], h \in\{1,2,3\}$, are chosen such that $\left[\bar{v}_{1}\left(t_{1}\right)+\bar{v}_{2}\left(t_{2}\right)+\bar{v}_{3}\left(t_{3}\right)\right]^{T} c=0$ and

$$
\bar{\beta}_{h}\left(t_{h}\right) \leq \sum_{j \in\{1,2,3\}, j \neq h} \bar{\beta}_{j}\left(t_{j}\right) \quad \text { holds for } h \in\{1,2,3\}
$$

then after having squeezed the subgraphs sufficiently we can employ Obs. 10 in order to find an equilibrium in the plane spanned by $d_{1}, d_{2}, d_{3}$. In fact, if equality holds for 


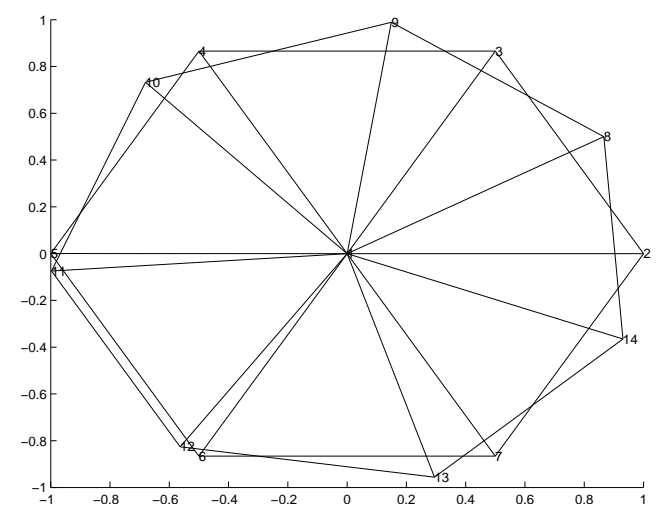

Figure 12: Optimal two dimensional embedding of two wheels with identical hub, see Rem. 16. The construction of the proof of Th. 15 (i) would yield a three dimensional embedding.

one of the three relations then the updated three vectors $d_{1}^{\prime}, d_{2}^{\prime}, d_{3}^{\prime}$ will only span a one dimensional subspace and we are done. In order to take care of the last direction $c$, let $\tau\left(t_{1}\right)$ be a nondecreasing continuous function satisfying $\left[\bar{v}_{1}\left(t_{1}\right)+\bar{v}_{2}\left(\tau\left(t_{1}\right)\right)+\bar{v}_{3}\left(\tau\left(t_{1}\right)\right]^{T} c=\right.$ 0 for small enough $t_{1} \in[0,1]$ so that $\tau\left(t_{1}\right) \leq 1$. Such a function exists as $\bar{v}_{1}(t)^{T} c$ is strictly decreasing in $t$ (because $\left.\bar{\beta}_{1}(0)>0\right),\left(\bar{v}_{2}(t)+\bar{v}_{3}(t)\right)^{T} c$ is strictly increasing in $t$, and $\left[\bar{v}_{1}(0)+\bar{v}_{2}(0)+\bar{v}_{3}(0)\right]^{T} c=0$. Let $\bar{t}_{1}$ be the smallest $t_{1} \in[0,1]$ such that (6) with $t_{2}=t_{3}=\tau\left(t_{1}\right)$ does not hold strictly for all $h \in\{1,2,3\}$. Such $\bar{t}_{1} \in[0,1]$ exists, as (6) must hold strictly for $t_{1}=t_{2}=t_{3}=0$ (or the dimension would be $|S|$ already) and $\bar{\beta}_{h}(1)=0$ for all $h \in\{1,2,3\}$. Therefore applying Obs. 10 to $v_{i}^{\prime \prime}=v_{i}^{\prime}\left(\bar{t}_{1}\right), i \in H_{1}^{\prime}$ and $v_{i}^{\prime \prime}=v_{i}^{\prime}\left(\tau\left(\bar{t}_{1}\right)\right), i \in H_{h}^{\prime}$ for $h \in\{2,3\}$ yields a feasible and optimal embedding of dimension $|S|$.

Remark 16 A solution corresponding to the modified solution of the theorem is not necessarily an optimal embedding of minimal dimension. Consider, e.g., the graph consisting of two wheels with identical hub and rims of $k$ and $k+1$ nodes with $k \geq 6$, see Fig. 12 .

The structure of optimal embeddings seems to be tightly related to tree-decompositions, whose definition we recall next.

Definition 17 For a graph $G=(N, E)$ let $T=(\mathcal{N}, \mathcal{E})$ be a tree with $\mathcal{N} \subseteq 2^{N}$ and $\mathcal{E} \subseteq\left(\begin{array}{c}\mathcal{N} \\ 2\end{array}\right)$ satisfying the following requirements:

(i) $N=\bigcup_{U \in \mathcal{N}} U$.

(ii) For every $e \in E$ there is a $U \in \mathcal{N}$ with $e \subseteq U$.

(iii) If $U_{1}, U_{2}, U_{3} \in \mathcal{N}$ with $U_{2}$ on the $T$-path from $U_{1}$ to $U_{3}$, then $U_{1} \cap U_{3} \subseteq U_{2}$.

Then $T$ is called a tree-decomposition of $G$. The width of $T$ is the number $\max \{|U|-1$ : $U \in \mathcal{N}\}$. The tree-width $t w(G)$ is the least width of any tree-decomposition of $G$.

Note, that in a tree decomposition $T=(\mathcal{N}, \mathcal{E})$ of $G=(N, E)$, any $U \in \mathcal{N}$ and any $U \cap U^{\prime}$ with $\left\{U, U^{\prime}\right\} \in \mathcal{E}$ is a separator of $G$ (see e.g. Lemma 12.3.1 in [5]). The next lemma asserts, 
that for any optimal embedding any tree-decomposition has "central nodes" containing the origin in their convex hull.

Lemma 18 Let $T=(\mathcal{N}, \mathcal{E})$ be a tree decomposition of a connected graph $G=(N, E)$ and $v_{i} \in \mathbb{R}^{n}, i \in N$, an optimal embedding of (4). Then there is at least one node $S \in \mathcal{N}$ with $0 \in \operatorname{conv}\left\{v_{s}: s \in S\right\}$, i.e., the convex hull of the points of $S$ contains the origin.

Proof. In order to show that there exists a node $S \in \mathcal{N}$ with $0 \in \mathcal{S}=\operatorname{conv}\left\{v_{s}: s \in S\right\}$ consider a smallest subtree $T^{\prime}=\left(\mathcal{N}^{\prime}, \mathcal{E}^{\prime}\right)$ of $T$ with $0 \in \operatorname{conv}\left\{v_{i}: i \in \bigcup_{U \in \mathcal{N}^{\prime}} U\right\}$. Such a tree exists since the condition holds for $T^{\prime}=T$. Let the convex combination giving the origin be described by $K \subset \bigcup_{U \in \mathcal{N}^{\prime}} U$ and $\alpha \in \mathbb{R}_{+}^{K}$ with $\alpha^{T} e=1$ so that $\sum_{i \in K} \alpha_{i} v_{i}=0$.

Assume, for contradiction, that $\left|\mathcal{N}^{\prime}\right|>1$ and Then there is an edge $\left\{U, U^{\prime}\right\} \in \mathcal{E}^{\prime}$ and $S^{\prime}=U \cap U^{\prime}$ is a separator of $G$. Deleting edge $\left\{U, U^{\prime}\right\}$ from $T^{\prime}$ splits $T^{\prime}$ into two nonempty subtrees $T_{j}^{\prime}=\left(\mathcal{N}_{j}^{\prime}, \mathcal{E}_{j}^{\prime}\right)$ for $j \in\{1,2\}$ with $0 \notin \operatorname{conv}\left\{v_{i}: i \in \bigcup_{U \in \mathcal{N}_{j}^{\prime}} U\right\}$ by assumption. Set $N_{j}^{\prime}=\bigcup_{U \in \mathcal{N}_{j}^{\prime}} U$. Because $S^{\prime} \subset N_{j}^{\prime}$ for $j \in\{1,2\}$ we obtain $0 \notin \mathcal{S}^{\prime}=\operatorname{conv}\left\{v_{i}: i \in S^{\prime}\right\}$. Thus, the separator-shadow Th. 12 applied to $S^{\prime}$ implies that, w.l.o.g., for all $i \in N_{1}^{\prime}$, $\left[v_{i}, 0\right] \cap \mathcal{S}^{\prime} \neq \emptyset$. Set $K_{1}=K \cap\left(N_{1}^{\prime}-S^{\prime}\right), K_{2}=K \backslash K_{1} \subseteq N_{2}^{\prime}$ and, for $j \in\{1,2\}$, $\bar{\alpha}_{j}=\sum_{i \in K_{j}} \alpha_{i}$ and $\bar{v}_{j}=\frac{1}{\bar{\alpha}_{j}} \sum_{i \in K_{j}} \alpha_{i} v_{i} \in \operatorname{conv}\left\{v_{i}: i \in N_{j}\right\}$. Then $0=\bar{\alpha}_{1} \bar{v}_{1}+\bar{\alpha}_{2} \bar{v}_{2} \in\left[\bar{v}_{1}, \bar{v}_{2}\right]$ and $\emptyset \neq \mathcal{S}^{\prime} \cap\left[\bar{v}_{1}, 0\right] \subset\left[\bar{v}_{1}, \bar{v}_{2}\right]$, so there is a $p \in \mathcal{S}^{\prime} \subset \operatorname{conv}\left\{v_{i}: i \in N_{2}^{\prime}\right\}$ with $0 \in\left[p, \bar{v}_{2}\right] \subset$ $\operatorname{conv}\left\{v_{i}: i \in N_{2}^{\prime}\right\}$, a contradiction. Hence, $T^{\prime}$ consists of only one node.

Theorem 19 Let $G=(N, E)$ be a connected graph, then there exists an optimal embedding for (4) of dimension at most $t w(G)+1$.

Proof. Let $T=(\mathcal{N}, \mathcal{E})$ be a tree decomposition of $G=(N, E)$ and assume, w.l.o.g., that all nodes $U \in \mathcal{N}$ are distinct subsets of $N$. Note, that for any optimal embedding $v_{i}$ the set of nodes $S \in \mathcal{N}$ with $0 \in \operatorname{conv}\left\{v_{i}: i \in S\right\}$ (called a zero-node in the sequel) and the set of edges $\left\{S, S^{\prime}\right\} \in \mathcal{E}$ with $0 \in \operatorname{conv}\left\{v_{i}: i \in S \cap S^{\prime}\right\}$ (called a zero-edge) form a nonempty subtree of $T$ (the zero-subtree) due to the separator-shadow Th. 12 and L. 18. Starting with a leaf of the current zero-subtree, we will walk off in "inward" direction, splitting the tree alternatingly with zero-nodes and zero-edges into one part containing visited nodes and one or several unvisited parts while ensuring all the time, that the part of the tree, that contains visited nodes is in dimension bounded by the size of the node incident to the separator.

For $r=0,1, \ldots$ and an $S^{r} \in \mathcal{N} \cup\left\{U \cap U^{\prime}:\left\{U, U^{\prime}\right\} \in \mathcal{E}\right\}$ (actually it will hold that $S^{r} \in \mathcal{N}$ whenever $r$ even and $S^{r}=U \cap U^{\prime}$ when $r$ is odd) we will use the following conventions. Removing $S^{r}$ from $T$, i.e. removing the corresponding edge or node of $T$, splits $T$ into connected components $T_{j}^{r}=\left(\mathcal{N}_{j}^{r}, \mathcal{E}_{j}^{r}\right)$ for $j \in M^{r}=\left\{1, \ldots, m^{r}\right\}$. We set $K_{j}^{r}=\bigcup_{U \in N_{j}^{r}} \backslash S^{r}$ for $j \in M^{r}$ and let $U_{j}^{r} \in \mathcal{N}_{j}$ denote the unique adjacent/incident neighbor node of $T_{j}^{r}$ to $S^{r}$ in $T$. So $S^{r}$ is a separator of $G$ inducing a partition $\left(S^{r}, K_{1}^{r}, \ldots, K_{m^{r}}^{r}\right)$ of $N$.

Let $v_{i}^{0}, i \in N$ be an initial optimal embedding and let $S^{0}$ be a leaf of the corresponding zero-tree. If for all neighbors $U_{j}^{0}, j \in M^{0}$, it holds that $0 \notin \operatorname{conv}\left\{v_{i}^{0}: i \in S^{0} \cap U_{j}^{0}\right\}$ then 
the separator-shadow Th. 12 asserts, that the embedding has dimension $\operatorname{dim} \mathcal{S}^{0} \leq\left|S^{0}\right|-1$ (one less than the cardinality, since the origin is contained in the convex hull) and we are done. Otherwise there is unique neighbor zero-node $U_{h}^{0}$ with $h \in M^{0}$ with $0 \in \operatorname{conv}\left\{v_{i}^{0}\right.$ : $\left.i \in S^{0} \cap U_{h}^{0}\right\}$. Set $r=1, v_{i}^{r}=v_{i}^{0}, i \in N$ and $S^{r}=S^{0} \cap U_{h}^{0}$.

Step 1 ( $r$ is odd): For the separator $S^{r}$ (an edge) in $T$, let $j^{r} \in M^{r}$ be the index of the tree $T_{j^{r}}=\left(\mathcal{N}_{j^{r}}^{r}, \mathcal{E}_{j^{r}}^{r}\right)$ with $S^{r-1} \in \mathcal{N}_{j^{r}}^{r}$. Observe, that for the current embedding $v_{i}^{r}, i \in N$, there is a $\bar{b}$ so that $v_{i} \in \operatorname{span}\left\{v_{i}^{r}: i \in S^{r-1}\right\}+\{\beta \bar{b}: \beta \geq 0\}$ for $i \in K_{j^{r}}^{r}$ (for $r=1, S^{0}$ is a leaf of the zero-tree of $v_{i}^{r}$ and employing the separator-shadow Th. 12 the claim holds for an arbitrary direction $\bar{b}$ orthogonal to $\left.\operatorname{span}\left\{v_{i}^{r}: i \in S^{r-1}\right\}\right)$ and $0 \in \operatorname{conv}\left\{v_{i}^{0}: i \in S^{r}\right\}$.

Apply Th. 15 (for $v_{i}=v_{i}^{r}, i \in N$ and $S=S^{r}$ with partitioning $K_{j}^{r}$ for $j \in M=M^{r}$ ) and first assume that case (ii) holds. Let $v_{i}^{\prime}, i \in N$ be the embedding given there and observe that its dimension is at most $\left|S^{r}\right|+1$. Since $S^{r}=U \cap U^{\prime}$ for some $\left\{U, U^{\prime}\right\} \in \mathcal{E}$ one of $U$ and $U^{\prime}$ has cardinality at least $\left|S^{r}\right|+1$, so the embedding $v_{i}^{\prime}, i \in N$ satisfies the required bound.

Next assume that case (i) of Th. 15 holds. Let $v_{i}^{\prime}(i \in N), \mathcal{L}, b_{j} \hat{\jmath} \in M^{r}$ be the objects specified there. If $\hat{\jmath}=j^{r}$ then $v_{i}^{\prime} \in \operatorname{span}\left\{v_{i}^{\prime}, h: i \in S^{r-1}\right\}$ (since $S^{r} \subseteq S^{r-1}$ and we found an optimal embedding of the required dimension. If $\hat{\jmath} \neq j^{r}$, observe that all nodes of the "visited" subtree $T_{j^{r}}$ are embedded in a flat halfspace, $v_{i}^{\prime} \in \mathcal{L}+\left\{\beta \sum_{i \in K_{\hat{\gamma}}} v_{i}^{\prime}: \beta \geq 0\right\}$ for $i \in K_{j^{r}}^{r} \cup S^{r}$. Set $v_{i}^{r+1}=v_{i}^{\prime}, S^{r+1}=U_{\hat{\jmath}}^{r}$ increase $r$ by one and continue with Step 2 .

Step 2 ( $r$ is even): Like in Step 1, let, for the separator $S^{r}$ (a node) in $T, j^{r} \in M^{r}$ be the index of the tree $T_{j^{r}}=\left(\mathcal{N}_{j^{r}}^{r}, \mathcal{E}_{j^{r}}^{r}\right)$ with $S^{r-2} \in \mathcal{N}_{j^{r}}^{r}$. Then for the current embedding $v_{i}^{r}, i \in N$ there is a $\bar{b}$ so that $v_{i} \in \operatorname{span}\left\{v_{i}^{r}: i \in S^{r-1}\right\}+\{\beta \bar{b}: \beta \geq 0\}$ for $i \in K_{j^{r}}^{r}$ (see the foregoing Step 1, case (i) with $\hat{\jmath} \neq j^{r}$ ) and $0 \in \operatorname{conv}\left\{v_{i}^{0}: i \in S^{r}\right\}$, because $S^{r-1} \subset S^{r}$.

Apply Th. 15 (for $v_{i}=v_{i}^{r}, i \in N$ and $S=S^{r}$ with partitioning $K_{j}=K_{j}^{r}$ for $j \in M=$ $M^{r}$ ) and this time first assume that case (i) holds. Let $v_{i}^{\prime}(i \in N), \mathcal{L}, \hat{\jmath} \in M^{r}$ be the objects specified there. If $\hat{\jmath}=j^{r}$, then, making use of $h=0$ because of $\bar{b}$ in Th. 15 (ii), the embedding $v_{i}^{\prime}$ is contained in $\mathcal{L}+\{\beta \bar{b}: \beta \in \mathbb{R}\}\left(\left\{v_{i}^{r}: i \in S^{r-1}\right\} \in \mathcal{L}\right.$ by $\left.S^{r-1} \subset S^{r}\right)$, so the dimension is at most $\left|S^{r}\right|$. If $\hat{\jmath} \neq j^{r}$, then $\left\{S^{r}, U_{\hat{\jmath}}^{r}\right\} \in \mathcal{E}$, set $S^{r+1}=S^{r} \cap U_{\hat{\jmath}}^{r}, v_{i}^{r+1}=v_{i}^{\prime}$ for $i \in N$. Observe that $0 \in \operatorname{conv}\left\{v_{i}^{r+1}: S^{r+1}\right.$ because otherwise the separator-shadow Th. 12 would imply $\beta_{\jmath}=0$ in (ii). Furthermore, all nodes of the "visited" subtree $T_{j^{r}}$ and $S^{r}$ are embedded in a flat halfspace, $v_{i}^{r+1} \in \mathcal{L}+\left\{\beta \sum_{i \in K_{\hat{\jmath}}} v_{i}^{r+1}: \beta \geq 0\right\}$ for $i \in K_{j^{r}}^{r} \cup S^{r}$. Increase $r$ by one and go to Step 1.

Because $T$ contains no cycles and only finitely many vertices, the process must either stop at some point, or, finally, meet case (ii) of Th. 15 and we show that we can stop then. Let $v_{i}^{\prime}(i \in N), \mathcal{L}, b_{j}\left(j \in M^{r}\right), d_{1}, d_{2}, d_{3}$ be the objects defined there. If $\operatorname{dim} \operatorname{span}\left\{v_{i}^{\prime}\right.$ : $i \in N\} \leq\left|S^{r}\right|$ or $S^{r}$ has a neighbor with $\left|S^{r}\right|+1$ nodes, then the $v_{i}^{\prime}, i \in N$ satisfy the requirements. Otherwise the $\left\{v_{i}^{\prime}: i \in S^{r}\right\}$ span an $\left|S^{r}\right|-1$ dimensional simplex and for each neighbor $U_{j}^{r}, j \in M^{r}$, it holds that $\left|U_{h}^{r} \cap S^{r}\right|=\left|S^{r}\right|-1$. This is case (iii) of Th. 15 and the proof is complete.

Remark 20 An optimal embedding of (4) computed by interior point methods will in general have maximal dimension instead of minimal dimension due to the properties of the 
central path (e.g., the computed solution for a star on $n$ nodes will put the $n-1$ degree one nodes as the vertices of an $n-2$ dimensional simplex while the minimal dimension would be either one for $n$ odd or two for $n$ even). This may be undesirable in the context of potential applications as indicated in Remark 14. In this case the proof of Th. 19 provides an algorithm for constructing for any given tree-decomposition $T=(\mathcal{N}, \mathcal{E})$ of $G$ and any optimal embedding $v_{i}, i \in N$ of (4) an optimal embedding $v_{i}^{\prime}$ satisfying $\operatorname{dim} \operatorname{conv}\left\{v_{i}^{\prime}: i \in\right.$ $N\} \leq \max \{|U|: u \in \mathcal{N}\}$. Even though it is NP-complete to find a tree-decomposition of width $\operatorname{tw}(G)$, there is still good hope to end up with a low dimensional embedding, because the proof also illustrates, that the bound on the dimension mainly depends on the size of a central separating node in the tree-decomposition, and its size may be significantly smaller than the tree-width.

\section{Some Special Cases and Examples}

We first present, as examples, the complete graphs and then a class of graphs for which the bound of Th. 19 is attained.

Example 21 (Complete Graphs) For $K_{n}=(\{1, \ldots, n\},\{\{i, j\}: 1 \leq i<j \leq n\})$ we show that the unique optimal embedding is the regular $n-1$ dimensional simplex with all points lying on the ball of radius $r_{n}=\sqrt{\frac{n-1}{2 n}}$. The optimal $X$ is given by $X_{i i}=r_{n}^{2}=\frac{n-1}{2 n}$ for $1 \leq i \leq n, X_{i j}=X_{j i}=-\frac{r_{n}^{2}}{n-1}=-\frac{1}{2 n}$ for $1 \leq i<j \leq n$, and the optimal weights are $w_{i j}=\frac{1}{n}$ for $1 \leq i<j \leq n$. Choosing $\mu=\frac{1}{n}$ we compute $L_{w}+\mu e e^{T}-I=0$, so $(w, \mu)$ is feasible for (2) with objective $\frac{n-1}{2}$. Likewise, $X$ is feasible for (3) and $\langle I, X\rangle=\frac{n-1}{2}$, so optimality is shown. Furthermore, since $w_{i j}>0$ for all $i j$, the constraints $\left\langle E_{i j}, X\right\rangle=1$ hold for all optimal $X$, i.e., the embedding must have all points pairwise at distance one. So the regular $n-1$ dimensional simplex is the only optimal embedding. Note that the tree-width of $K_{n}$ is $n-1$, thus the complete graphs are not tight with respect to the bound of Th. 19 .

Example 22 (Graphs with tight dimension bound) We append to $K_{n}$ three independent vertices that are completely linked to $K_{n}$ resulting in a graph $G(n)=(\{1, \ldots, n+$ $3\}, E(n)=\{\{i, j\}: 1 \leq i \leq n, i<j \leq n+3\})$. The tree-width of $G(n)$ is $n$ and for $n \geq 4$ the minimal dimension of an optimal embedding of $G(n)$ is $n+1$. In fact, we show that, for $n \geq 4$, the vertices of $K_{n}$ are again arranged as a centrally symmetric $n-1$ dimensional simplex with all points lying on a ball of radius $r_{n}=\sqrt{\frac{n-1}{2 n}}$, while the three new points are arranged centrally symmetric on a circle orthogonal to this simplex with radius $\bar{r}=\sqrt{\frac{n+1}{2 n}}$. The optimum of (3) is obtained by extending the optimum of Ex. 21 with $X_{i i}=\bar{r}^{2}=\frac{n+1}{2 n}$ for $n<i \leq n+3, X_{i j}=X_{j i}=-\frac{\bar{r}^{2}}{2}=-\frac{n+1}{4 n}$ for $n<i<j \leq n+3$, and $X_{i j}=X_{j i}=0$ for $1 \leq i \leq n, n<j \leq n+3$. The optimal weights are $w_{i j}=\frac{1}{n}$ for $1 \leq i \leq n, n<j \leq n+3$ and $w_{i j}=\frac{1}{n}-\frac{3}{n^{2}}$ for $1 \leq i<j \leq n$ (use Rem. 1 and symmetry). Setting $\mu=\frac{1}{n}$ the slack 


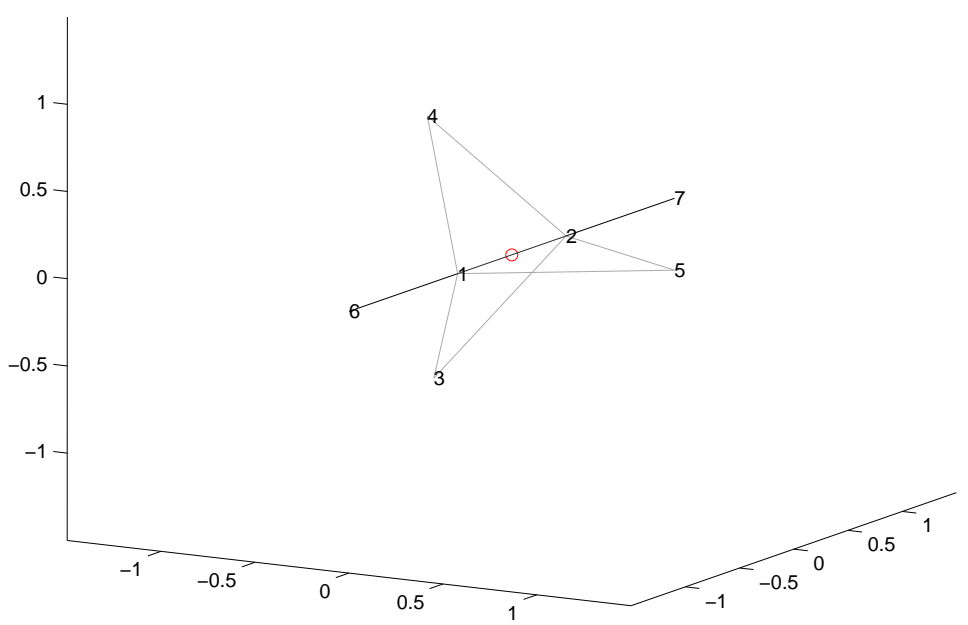

Figure 13: A graph with tree-width 2 and optimal embedding of dimension at least three, see Ex. 22 (the central circle indicates the origin).

matrix of (2) computes to

$$
Z=L_{w}+\frac{1}{n} e e^{T}-I=\left[\begin{array}{cc}
\frac{3}{n^{2}} J_{n} & 0 \\
0 & \frac{1}{n} J_{3}
\end{array}\right] \succeq 0
$$

where $J_{k}$ denotes the square matrix of all ones of order $k$. Therefore $(w, \mu)$ is feasible for (2), the objective value is $3 n \frac{1}{n}+\frac{n(n-1)}{2}\left(\frac{1}{n}-\frac{3}{n^{2}}\right)=\frac{3}{2}(n+1)+\frac{n-1}{2}$. Likewise, $X$ is positive semidefinite because it is a gram matrix. Furthermore, $X$ satisfies all distance constraints and has the same objective value. Hence the primal and the dual solution are optimal.

Now take any optimal embedding $v_{i}, i=1, \ldots, n$ and set $V=\left[v_{1}, \ldots, v_{n}\right]$. Since $w>0$, all optimal embeddings must have all edge lengths equal to one, $\left\|v_{i}-v_{j}\right\|=1$ for all $i j \in E(n) . B y(7)$ and semidefinite complementarity it holds that $\left\langle V^{T} V, Z\right\rangle=0$, thus $\sum_{i=1}^{n} v_{i}=0$ and $\sum_{i=n+1}^{n+3} v_{i}=0$. So the embedding of $K_{n}$ must be centrally symmetric like in Ex. 21, and by the distance constraints each of the three additional vertices must be embedded orthogonal to the embedding of $K_{n}$ with distance $\bar{r}$ to the origin. As the three vectors have to sum up to zero, this can only be done in two additional dimensions. This completes the proof.

For $n=1$ the construction yields a star with one central and three exterior nodes and the bound is also tight. For $n=2$ the embedding described above is not optimal (it would collapse to the image of the star), for $n=3$ the embedding is optimal but not of minimal dimension. Without going into details, the cases $n=2,3$ can be extended to tight examples by appending to each node of $K_{n}$ yet another node by a single edge, see Fig. 13 for an illustration of the resulting embedding for $n=2$. 
In spite of these examples, the bound of Th. 19 seems far too pessimistic for many important graph classes. E.g., for planar graphs the tree-width can be made arbitrarily large but we have not succeeded in exhibiting an example whose minimal dimension exceeds three.

We turn to the special structure of the embedding if the separator is a single node.

Definition 23 In a graph $G=(N, E)$ a node $\hat{\imath} \in N$ is called an articulation point (or cut vertex) if deleting $\hat{\imath}$ and all its incident edges increases the number of connected components in the graph.

For a connected graph $G=(N, E)$ with articulation point $\hat{\imath}$ let $G_{j}^{\prime}=\left(N_{j}^{\prime}, E_{j}^{\prime}\right), j=$ $1, \ldots, m$ denote the connected components arising from $G$ by deleting $\hat{\imath}$ and let $G_{j}=G\left(N_{j}^{\prime} \cup\right.$ $\{\hat{\imath}\})$ be the induced subgraphs of $G$ arising from adjoining $\hat{\imath}$ to $N_{j}^{\prime}$. Then we call $G_{j}=$ $\left(N_{j}, E_{j}\right), j=1, \ldots, m, a$ splitting of $G$ in $\hat{\imath}$.

$A$ breadth first search tree $T$ (BFS-tree) of a connected graph $G=(N, E)$ with respect to $\hat{\imath} \in N$ is a spanning tree so that the distance from $\hat{\imath}$ to $j$ is the same in $G$ and $T$ for all $j \in N$.

For articulation points the separator-shadow Th. 12 admits the following corollary.

Corollary 24 Let $G=(N, E)$ be a connected graph with articulation point $\hat{\imath}$ and splitting $G_{j}=\left(N_{j}, E_{j}\right), j \in M=\{1, \ldots, m\}$, in $\hat{\imath}$. Let $v_{i} \in \mathbb{R}^{n}, i \in N$, be an optimal embbeding of (4) with $v_{\hat{\imath}} \neq 0$. Then there is a $\hat{\jmath} \in M$ so that for all $j \in M \backslash\{\hat{\jmath}\}$ and $i \in N_{j}$ it holds that $v_{i}=v_{\hat{\imath}}+d_{G}(\hat{\imath}, i) \frac{v_{\hat{\imath}}}{\left\|v_{\hat{\imath}}\right\|}$, where $d_{G}(\cdot, \cdot)$ denotes the distance in $G$.

In words, the embedding of the nodes of $G_{j}, j \in M \backslash\{\hat{\jmath}\}$ described in the theorem corresponds to stretching the BFS-tree of $G_{j}$ with respect to $\hat{\imath}$ as far as possible into the direction $\frac{v_{\hat{\imath}}}{\left\|v_{\hat{\imath}}\right\|}$.

Proof. By the equilibrium constraint and $v_{\hat{\imath}} \neq 0$ there must be at least one $\hat{\jmath} \in M$ for which the statement does not hold. The separator-shadow Th. 12 implies that the embeddings of all other $j \in M \backslash\{\hat{\jmath}\}$ must lie on the half ray $\left\{v_{\hat{\imath}}+\lambda \frac{v_{\hat{\imath}}}{\left\|v_{\hat{\imath}}\right\|}: \lambda \geq 0\right\}$ (for each $h \in M \backslash\{\hat{\jmath}\}$ successively pick $S=\{\hat{\imath}\}, K_{1}=N_{h} \backslash S$ and $\left.K_{2}=\bigcup_{j \in M \backslash\{h\}} N_{j} \backslash S\right)$.

The embedding of the nodes of $G_{h}, h \in M \backslash\{\hat{\jmath}\}$, described in the theorem satisfies the distance constraints, because each edge $i j \in E_{h}$ implies that $\left|d_{G}(\hat{\imath}, i)-d_{G}(\hat{\imath}, j)\right| \leq d_{G}(i, j)=$ 1 by the triangle inequality. Suppose, for contradiction, that there is an $h \in M \backslash\{\hat{\jmath}\}$ with the embedding $v_{i}, i \in N_{h}$, not of this form. Let $K=\left\{i \in N_{h}:\left\|v_{i}-v_{\hat{\imath}}\right\|<d_{G}(\hat{\imath}, i)\right\}$ and set $d=$ $\frac{v_{\hat{\imath}}}{\left\|v_{\hat{\imath}}\right\|} \min _{i \in K}\left\{d_{G}(\hat{\imath}, i)-\left\|v_{i}-v_{\hat{\imath}}\right\|\right\}$. Then we can translate all points in $K$ by $d$ without violating the distance constraints. Combining this translation with recentering yields a new feasible embedding $v_{i}^{\prime}=v_{i}+\frac{|N|-|K|}{|N|} d$ for $i \in K$ and $v_{i}^{\prime}=v_{i}-\frac{|K|}{|N|} d$ for $i \in N \backslash K$. By Obs. 4 (use $\bar{v}_{K}=\frac{1}{|K|} \sum_{i \in K} v_{i} \in\left\{v_{\hat{\imath}}+\lambda \frac{v_{\hat{\imath}}}{\left\|v_{\hat{\imath}}\right\|}: \lambda \geq 0\right\}$ and $\left.\bar{v}_{N \backslash K}=-\frac{|K|}{|N|-|K|} \bar{v}_{K}\right)$ the translations increase the objective value by $|K|\left\langle 2 \bar{v}_{K}+\frac{|N|-|K|}{|N|} d, \frac{|N|-|K|}{|N|} d\right\rangle+|N-K|\left\langle 2 \bar{v}_{N \backslash K}-\frac{|K|}{|N|} d,-\frac{|K|}{|N|} d\right\rangle>0$. Therfore the $v_{i}$ could not have been optimal, a contradiction.

For a given articulation point we next give precise criteria for recognizing those subgraphs in the splitting that can always be embedded on a ray in an appropriate optimal 
embedding. In order to give a more intuitive formulation we prepare the ground by giving names to the objects of interest.

Definition 25 Let $G=(N, E)$ be a connected graph with articulation point $\hat{\imath}$ and splitting $G_{j}=\left(N_{j}, E_{j}\right), j \in M=\{1, \ldots, m\}$ in $\hat{\imath}$. Set $\beta_{j}=\sum_{i \in N_{j}} d_{G}(\hat{\imath}, i)$. We call $\hat{\imath}$ central if $\beta_{h} \leq \sum_{j \in M \backslash\{h\}} \beta_{j}$ for all $h \in M$. If $\beta_{h}>\sum_{j \in M \backslash\{h\}} \beta_{j}$ for some $h \in M$ then call $G_{h}$ the heavy subgraph split off by $\hat{\imath}$. For a central articulation point the heavy subgraph is defined to be the empty graph.

Let $G=(N, E)$ be a connected graph and $G_{j}^{\prime}=\left(N_{j}^{\prime}, E_{j}^{\prime}\right), j \in M^{\prime}=\{1, \ldots, m\}$, a decomposition of $G$ into its blocks (bridges and maximal two-connected subgraphs). We call a block $G_{h}^{\prime}$ central if for each articulation point of $G$ the heavy subgraph contains $G_{h}^{\prime}$.

Let $\hat{\imath}$ be an articulation point of $G$ with $\hat{\imath} \in N_{h}^{\prime}$ for a block $G_{h}^{\prime}=\left(N_{h}^{\prime}, E_{h}^{\prime}\right)$ of $G$. If $G_{h}^{\prime}$ is not contained in the heavy subgraph split off by $\hat{\imath}$, then call $\hat{\imath}$ a root of block $G_{h}^{\prime}$.

We state the following two observations without proof.

Observation 26 Let $G_{h}$ be a block of a connected graph. $G_{h}$ has a root if and only if it is not central. If a root exists, it is unique.

Observation 27 A connected graph has a central block if and only if it has no central articulation point. Furthermore, the existing object is unique.

Corollary 28 Let $G=(N, E)$ be a connected graph with articulation point $\hat{\imath}$. There exists an optimal embedding $v_{i}, i \in N$, of (4) with the following property. Each subgraph of the associated splitting, that is not a heavy subgraph, is embedded on a one dimensional ray emanating from the origin and containing $v_{\hat{\imath}}$. If, in addition, $\hat{\imath}$ is central, then $v_{\hat{\imath}}=0$ in any optimal embedding of (4).

Proof. Let $G_{j}=\left(N_{j}, E_{j}\right), j \in M=\{1, \ldots, m\}$ be the splitting of $G$ in $\hat{\imath}$. Set $\beta_{j}=$ $\sum_{i \in N_{j}} d_{G}(\hat{\imath}, i)$. Let $v_{i}, i \in N$, be an arbitrary optimal embedding. If $v_{\hat{\imath}} \neq 0$ then Cor. 24 asserts the existence of a $\hat{\jmath} \in M$ with $\bar{v}=\sum_{i \in N_{\hat{\jmath}}} v_{i}=-\sum_{i \in N \backslash N_{\hat{\jmath}}}\left(v_{\hat{\imath}}+d(\hat{\imath}, i) \frac{v_{\hat{\imath}}}{\left\|v_{\hat{\imath}}\right\|}\right)=$ $-\left[\left(|N|-\left|N_{\hat{\jmath}}\right|\right) v_{\hat{\imath}}+\frac{v_{\hat{\imath}}}{\left\|v_{\hat{\imath}}\right\|} \sum_{j \in M \backslash\{\hat{\jmath}\}} \beta_{j}\right]$. Because $-\bar{v}^{T} \frac{v_{\hat{\imath}}}{\left\|v_{\hat{\imath}}\right\|}<\beta_{\hat{\jmath}}$ it follows that $\beta_{\hat{\jmath}}>\sum_{j \in M \backslash\{\hat{\jmath}\}} \beta_{j}$. For the case $v_{\hat{\imath}}=0$ it suffices to apply Th. 15 .

Using Th. 15 and similar arguments as in the proof of Cor. 24 one can show that an optimal embedding exists so that all blocks that are not central, are embedded on rays stretched out according to their BFS-tree with respect to their root. We give this result without proof.

Corollary 29 Let $G=(N, E)$ be a connected graph and $G_{j}=\left(N_{j}, E_{j}\right), j \in M=$ $\{1, \ldots, m\}$, a block decomposition of $G$. Then there exists an optimal embedding $v_{i} \in \mathbb{R}^{n}$, $i \in N$, of (4) with the following property. Let $G_{j}, j \in M$, be a block that is not central and let $r_{j} \in N_{j}$ denote its root, then $v_{i}=v_{r_{j}}+d_{G}\left(r_{j}, i\right) \frac{v_{r_{j}}}{\left\|v_{r_{j}}\right\|}$, for all $i \in N_{j}$ where $d_{G}(\cdot, \cdot)$ denotes the distance in $G_{j}$. Furthermore, if $G_{j}$ is a central block, then $0 \in \operatorname{conv}\left\{v_{i}: i \in N_{j}\right\}$ for any optimal embedding. 
In the case of trees, each block consists of a single edge and Cor. 29 takes the following special form.

Corollary 30 Let $v_{i} \in \mathbb{R}^{n}$ for $i \in N$ be an optimal solution of (4) for a tree $T=(N, E)$. In this embedding, either there exists a unique root node located at the origin, and each child lies at distance one from its parent on a ray emanating from the origin through its parent, or the origin is located in the interior of a straight line segment of length one between two (unique) adjacent nodes in $T$ and all nodes lie on the line spanned by these two nodes, having the same Euclidean distance to these two nodes as in the tree.

Remark 31 Cor. 30 may be used to construct an optimal embedding for trees directly without the need of solving a semidefinite program. Note, that the absolute algebraic connectivity was already determined for trees in [8] and Cor. 30 offers a new dual view of these results.

\section{References}

[1] D. Bertsimas and Y. Ye. Semidefinite relaxations, multivariate normal distributions, and order statistics. In D.-Z. Du and P. Pardalos, editors, Handbook of Combinatorial Optimization, pages 1-19. Kluwer Academic Publishers, 1998.

[2] K. L. Boyer and K. Sengupta. Modelbase partitioning using property matrix spectra. Computer Vision and Image Understanding, 70(2):177-196, May 1998.

[3] T. F. Chan, P. Ciarlet, and W. K. Szeto. On the optimality of the median cut spectral bisection graph partitioning method. SIAM J. Sci. Comput., 18(3):943-948, May 1997.

[4] D. Cvetković, M. Doob, and H. Sachs. Spectra of Graphs. Theory and application. J. A. Barth Verlag, Leipzig, 3rd edition, 1995.

[5] R. Diestel. Graph Theory. Springer, Berlin, 2nd edition, 2000.

[6] M. Fiedler. Algebraic connectivity of graphs. Czechoslovak Mathematical Journal, 23:298-305, 1973.

[7] M. Fiedler. Laplacian of graphs and algebraic connectivity. Combinatorics and Graph Theory, 25:57-70, 1989.

[8] M. Fiedler. Absolute algebraic connectivity of trees. Linear and Multilinear Algebra, 26:85-106, 1990.

[9] M. Fiedler. A geometric approach to the Laplacian matrix of a graph. IMA Vol. Math. Appl., 50:73-98, 1993.

[10] M. Fiedler. Some minimax problems for graphs. Discrete Mathematics, 121:65-74, 1993. 
[11] M. X. Goemans and D. P. Williamson. Improved approximation algorithms for maximum cut and satisfiability problems using semidefinite programming. J. ACM, 42:1115-1145, 1995.

[12] C. Helmberg, B. Mohar, S. Poljak, and F. Rendl. A spectral approach to bandwidth and separator problems in graphs. Linear and Multilinear Algebra, 39:73-90, 1995.

[13] X. Ji and H. Zha. Extracting shared topics of multiple documents. Lect. Notes Comput. Sci., 2637:100-110, 2003.

[14] M. Juvan and B. Mohar. Laplace eigenvalues and bandwidth-type invariants of graphs. J. Graph Theory, 17(3):393-407, 1993.

[15] A. Kaveh and H. A. Rahimi Bondarabady. Bisection for parallel computing using Ritz and Fiedler vectors. Acta Mechanica, 167:131-144, Feb. 2004.

[16] J. Keuchel, C. Schnörr, C. Schellewald, and D. Cremers. Binary partitioning, perceptual grouping, and restoration with semidefinite programming. IEEE Transactions on Pattern Analysis and Machine Intelligence, 25(11):1364-1379, Nov. 2003.

[17] L. Lovász. On the Shannon capacity of a graph. IEEE Transactions on Information Theory, IT-25(1):1-7, Jan. 1979.

[18] B. Mohar. The Laplacian spectrum of graphs. In Graph Theory, Combinatorics, and Applications, pages 871-898. John Wiley and Sons, 1991.

[19] B. Mohar. Graph Laplacians. In B. et al., editor, Topics in algebraic graph theory, volume 102 of Encyclopedia of Mathematics and Its Applications, pages 113-136. Cambridge University Press, 2004.

[20] B. Mohar and S. Poljak. Eigenvalues in combinatorial optimization. In Brualdi, Friedland, and Klee, editors, Combinatorial and Graph Theoretic Problems in Linear Algebra. IMA Volume in Mathematics and Applications, Vol. 50, 1993.

[21] A. Pothen, H. D. Simon, and K. Liou. Partitioning sparse matrices with eigenvectors of graphs. SIAM J. Matrix Anal. Appl., 11:430-452, 1990.

[22] H. D. Simon, A. Sohn, and R. Biswas. Harp: A dynamic sprectral partitioner. Journal of Parallel and Distributed Computing, 50:83-103, 1998.

[23] J. F. Sturm. Using SeDuMi 1.02, a MATLAB toolbox for optimization over symmetric cones (updated for version 1.05). Manual, Department of Econometrics, Tilburg University, P.O. Box 90153, 5000 LE Tilburg, The Netherlands, Oct. 2001.

[24] H. van der Holst, L. Lovász, and A. Schrijver. The Colin de Verdière graph parameter. In L. et al., editor, Graph theory and combinatorial biology, volume 7 of Bolyai Soc. Math. Stud., pages 29-85. János Bolyai Mathematical Society, Budapest, 1999. 
[25] H. Wolkowicz, R. Saigal, and L. Vandenberghe, editors. Handbook of Semidefinite Programming, volume 27 of International Series in Operations Research and Management Science. Kluwer Academic Publishers, Boston/Dordrecht/London, 2000.

[26] C. W. Wu. On Rayleigh-Ritz ratios of a generalized Laplacian matrix of directed graphs. Linear Algebra Appl., 402:207-227, 2005. 